\title{
On relations of the adjoint state to the value function for optimal control problems with state constraints
}

\author{
Hélène Frankowska and Marco Mazzola
}

\begin{abstract}
We consider an optimal control problem under state constraints and show that to every optimal solution corresponds an adjoint state satisfying the first order necessary optimality conditions in the form of a maximum principle and sensitivity relations involving the value function. Such sensitivity relations were recently investigated by P. Bettiol and R.B. Vinter for state constraints with smooth boundary. In the difference with their work, our setting concerns differential inclusions and nonsmooth state constraints. To obtain our result we derive neighboring feasible trajectory estimates using a novel generalization of the so-called inward pointing condition.
\end{abstract}

Mathematics Subject Classification (2010). Primary 49K15, 49Q12.

Keywords. Optimal control, Differential inclusion, State constraint, Maximum principle, Value function, Sensitivity, Co-state.

\section{Introduction}

Mathematical models arising in engineering and social sciences often involve nonautonomous dynamical systems depending on parameters. Typically restrictions are imposed on both parameters and states to respect all the model requirements. This leads to control systems and differential inclusions under state constraints. Trajectories of a given control system satisfying state constraints are called viable (in social sciences) or feasible (in engineering sciences). Their investigation attracted considerable attention. In the presence of state constraints control systems are difficult to deal with, because minor variations of an admissible control may drive trajectories out of the domain. One of the

Dedicated to Arrigo Cellina.

This work was co-funded by the European Union under the 7th Framework Programme "FP7-PEOPLE-2010-ITN", grant agreement number 264735-SADCO. 
topics that was intensively studied in the last decade concerns estimates on the distance of a given trajectory from the set of feasible trajectories, expressed in terms of the state constraint violation. In the literature, these results have been referred to as neighboring feasible trajectory (NFT) estimates.

Optimal control theory in the presence of state constraints has been developed since the late fifties. In particular, first order necessary conditions for optimality were derived in the form of a maximum principle in $[12,19]$. We refer to [23] and the bibliography contained therein for further results and comments on maximum principles for nonsmooth control systems and differential inclusions under state constraints. Another related topic under investigation since eighties concerns Hamilton-Jacobi equations under state constraints. For instance in [20] it was shown that the value function of an infinite horizon optimal control problem defined on the closure of an open set with smooth boundary is the unique (continuous) viscosity solution of the associated stationary Hamilton-Jacobi equation. To prove continuity of the value function a so called inward pointing condition (IPC) was used. Actually IPC implies that $K$ is the closure of its interior. Similarly, results of the present paper do not apply to state constraints whose interior is empty.

More precisely, let $F:[0,1] \times \mathbb{R}^{n} \leadsto \mathbb{R}^{n}$ be a set-valued map with compact nonempty images and $K$ be a nonempty closed subset of $\mathbb{R}^{n}$. Denote by $C_{K}(x)$ and $N_{K}(x)$ the Clarke tangent and normal cones to $K$ at $x \in K$. For every $t_{0} \in[0,1)$ and $x_{0} \in K$ consider the following differential inclusion

$$
\left\{\begin{array}{l}
x^{\prime}(t) \in F(t, x(t)) \quad \text { a.e. in }\left[t_{0}, 1\right] \\
x\left(t_{0}\right)=x_{0}
\end{array}\right.
$$

under state constraints of the form

$$
x(t) \in K \quad \forall t \in\left[t_{0}, 1\right] .
$$

Let $S_{\left[t_{0}, 1\right]}^{K}\left(x_{0}\right)$ be the set of all solutions to (1.1)-(1.2). If for some $L>0$ and for every trajectory $x(\cdot)$ of (1.1) with $x_{0} \in K$,

$$
\inf _{y \in S_{\left[t_{0}, 1\right]}^{K}\left(x_{0}\right)}\|x(\cdot)-y(\cdot)\|_{\infty} \leq L \max _{t \in\left[t_{0}, 1\right]} \operatorname{dist}(x(t), K),
$$

then (1.1)-(1.2) is said to enjoy NFT estimates in $\|\cdot\|_{\infty}$.

For Lipschitz $F$ and bounded $K$ having smooth boundary $\partial K$, NFT estimates follow from the classical IPC: for all $x \in \partial K$ and $t \in[0,1]$, there exists $w \in F(t, x)$ satisfying $\left\langle n_{x}, w\right\rangle<0$, where $n_{x}$ is the unit outward normal to $K$ at $x$ (see $[4,18]$ ). By the proof of Lemma 3.5 from Sect. 3 , for a continuous $F$, the classical IPC is equivalent to: for all $(t, x) \in[0,1] \times \partial K$,

$$
\forall v \in F(t, x) \text { with }\left\langle n_{x}, v\right\rangle \geq 0, \exists w \in F(t, x) \text { satisfying }\left\langle n_{x}, w-v\right\rangle<0 .
$$

A natural generalization of this condition is the following "nonsmooth" IPC which uses the notation $N_{K}^{1}(x):=N_{K}(x) \cap S^{n-1}$ : for all $(t, x) \in[0,1] \times \partial K$,

$$
\left\{\begin{array}{l}
\forall v \in F(t, x) \text { satisfying } \max _{n \in N_{K}^{1}(x)}\langle n, v\rangle \geq 0, \\
\exists w \in F(t, x) \text { satisfying } \max _{n \in N_{K}^{1}(x)}\langle n, w-v\rangle<0 .
\end{array}\right.
$$


In general (1.3) does not follow from the condition $F(t, x) \cap \operatorname{Int} C_{K}(x) \neq \emptyset$ or (equivalently) from $\exists w \in F(t, x)$ satisfying $\max _{n \in N_{K}^{1}(x)}\langle n, w\rangle<0$, which was used in [18] to get NFT estimates in $\|\cdot\|_{\infty}$ for Lipschitz $F$. For $F$ merely continuous with respect to time, this last condition is not sufficient for having NFT estimates (see [4] for a counter-example). Earlier counter-examples to NFT estimates with $F$ depending only on time in a discontinuous way can be found in [3]. It became clear then that a different inward pointing condition should be found. We proposed such new IPC in $[15,16]$. In the present paper we show that if $F$ is locally bounded around $[0,1] \times \partial K$, then our IPC takes a simpler, (1.3) like form. Then we apply our earlier results from [16] to derive NFT estimates in $W^{1,1}$ metric.

Let $g: \mathbb{R}^{n} \rightarrow \mathbb{R}$ and consider the Mayer optimal control problem

$$
\operatorname{minimize}\left\{g(x(1)) \mid x(\cdot) \in S_{[0,1]}^{K}\left(x_{0}\right)\right\} \text {. }
$$

It is well known that the classical Bolza problem of optimal control theory can be rewritten as the Mayer one via a simple change of variables. It augments the dimension of the state space by one and the state constraints become then $\mathbb{R} \times K$ instead of $K$. This new Mayer problem can be studied using results of the present work. In particular, because $N_{\mathbb{R} \times K}(r, x)=\{0\} \times N_{K}(x)$ for all $(r, x) \in \mathbb{R} \times K$, the IPC condition for the Bolza problem concerns only the set $K$. For the above reason we restrict our attention to the Mayer problem.

The associated value function $V:[0,1] \times \mathbb{R}^{n} \rightarrow \mathbb{R}$ is defined by

$$
V\left(t_{0}, y_{0}\right)=\inf \left\{g(x(1)) \mid x(\cdot) \in S_{\left[t_{0}, 1\right]}^{K}\left(y_{0}\right)\right\}
$$

with the convention $\inf \emptyset=+\infty$. The Hamiltonian $H:[0,1] \times \mathbb{R}^{n} \times \mathbb{R}^{n} \rightarrow \mathbb{R}$ is defined by $H(t, x, p)=\max _{v \in F(t, x)}\langle p, v\rangle$.

If $K=\mathbb{R}^{n}, g$ is differentiable and $F$ is given by a smooth control system, then with every optimal trajectory $\bar{x}(\cdot)$ of the Mayer problem we can associate an adjoint state $p(\cdot)$ (of the maximum principle) satisfying two relations

$$
(H(t, \bar{x}(t), p(t)),-p(t)) \in \partial_{+} V(t, \bar{x}(t)) \text { a.e. } \quad \text { and } \quad-p(t) \in\left(\partial_{+}\right)_{x} V(t, \bar{x}(t)) \forall t,
$$

where $\partial_{+} V(t, \bar{x}(t))$ (resp. $\left.\left(\partial_{+}\right)_{x} V(t, \bar{x}(t))\right)$ denotes the superdifferential of $V$ at $(t, \bar{x}(t))$ (resp. of $V(t, \cdot)$ at $\bar{x}(t))$, see $[6,7,21]$. These relations are referred to as sensitivity relations. For Lipschitz data similar results hold true with superdifferentials replaced by generalized gradients, see [11,24] and also [23] for an extension of the second sensitivity relation to unconstrained differential inclusions. Further results for differential inclusions under general state constraints can be found in $[8,9]$. In [5], in the context of control systems and smooth state constraints, NFT estimates combined with techniques of $[11,24]$ were applied in an astute way to deduce sensitivity relations with a particular type of subgradients of $V$. The maximum principle in [5] is expressed in terms of the Pontriagin Hamiltonian and [5, Theorem 3.2] concludes that two adjoint states can be found so that they satisfy each of two sensitivity relations separately.

In the present paper we consider a more general framework of differential inclusions and nonsmooth state constraints and prove the existence of an 
adjoint state satisfying both sensitivity relations for the same subgradients as those in [5].

The outline of the paper is as follows. In Sect. 2 we provide some preliminaries. Section 3 is devoted to NFT estimates, while Sect. 4 discusses their application to variational inclusions. The sensitivity relations are investigated in Sect. 5.

\section{Preliminaries}

We denote by $|\cdot|$ the Euclidean norm in $\mathbb{R}^{n}$, by $\langle\cdot, \cdot\rangle$ the inner product, by $S^{n-1}$ the unit sphere in $\mathbb{R}^{n}$, by $B$ the closed unit ball, and by $B\left(x_{0}, \varepsilon\right)$ and $\stackrel{\circ}{B}\left(x_{0}, \varepsilon\right)$ respectively the closed ball and the open ball of centre $x_{0}$ and radius $\varepsilon>0$. For a subset $A \subset \mathbb{R}^{n}$ let co $A$ states for the convex hull of $A$ and $\overline{c o} A$ for its closed convex hull. For every pair $(a, b) \in \mathbb{R}^{2}$, set $a \vee b=\max \{a, b\}$.

Definition 2.1. Let $f: \mathbb{R}^{n} \rightarrow \mathbb{R} \cup\{+\infty\}$ be an extended valued function and $x \in \mathbb{R}^{n}$ be so that $f(x)<\infty$. The contingent derivative of $f$ at $x$ in the direction $v \in \mathbb{R}^{n}$ is defined by

$$
D_{\uparrow} f(x)(v):=\liminf _{h \rightarrow 0+, v^{\prime} \rightarrow v} \frac{f\left(x+h v^{\prime}\right)-f(x)}{h}
$$

and the subdifferential of $f$ at $x$ by $\partial_{-} f(x)=\left\{p \in \mathbb{R}^{n} \mid\langle p, v\rangle \leq D_{\uparrow} f(x)(v) \forall v\right\}$. The limiting subdifferential of $f$ at $x$ is the set $\partial^{*} f(x):=\operatorname{Limsup}_{y \rightarrow x} \partial_{-} f(y)$, where Limsup denotes the Kuratowski upper limit.

Definition of limiting subdifferential given in [23, p.135] is equivalent to the above one thanks to [23, Proposition 4.4.3 and Theorem 4.6.3]. By [23, Proposition 4.7.6] for a locally Lipschitz around $x$ function $f, \operatorname{co}^{*} f(x)$ is equal to the Clarke generalized gradient $\partial f(x)$.

Let $G: \mathbb{R}^{m} \leadsto \mathbb{R}^{n}$ be a set-valued map having nonempty images. We say that $G$ is locally bounded at $x_{0}$ if there exists $\delta>0$ such that $\sup _{x \in B\left(x_{0}, \delta\right), v \in G(x)}|v|<\infty$ and that it is $\lambda$-Lipschitz on a set $A \subset \mathbb{R}^{m}$ if

$$
G\left(x_{1}\right) \subset G\left(x_{2}\right)+\lambda\left|x_{1}-x_{2}\right| B \quad \forall x_{1}, x_{2} \in A .
$$

Let $K$ be a nonempty closed subset of $\mathbb{R}^{n}$, Int $K$ be its interior and $\partial K$ its boundary, $d_{K}(x)=\operatorname{dist}(x, K)$ be the distance from $x \in \mathbb{R}^{n}$ to $K$. Denote by $C_{K}(x)$ and $N_{K}(x)$ respectively the Clarke tangent and normal cones to $K$ at $x \in K$. Recall that if $\operatorname{Int} C_{K}(x) \neq \emptyset$ for all $x \in \partial K$, then the set-valued maps $K \ni x \leadsto N_{K}(x) \cap S^{n-1}$ and $K \ni x \leadsto N_{K}(x) \cap B$ are upper semicontinuous. Define $N_{K}^{1}(x):=N_{K}(x) \cap S^{n-1}$ for all $x \in \partial K$.

For each $t_{0} \in[0,1]$ denote by $W^{1,1}\left(\left[t_{0}, 1\right] ; \mathbb{R}^{n}\right)$ the space of absolutely continuous functions $x:\left[t_{0}, 1\right] \rightarrow \mathbb{R}^{n}$, endowed with the norm $\|x(\cdot)\|_{W^{1,1}}=$ $\left|x\left(t_{0}\right)\right|+\int_{t_{0}}^{1}\left|x^{\prime}(t)\right| d t$ and by $\|x(\cdot)\|_{\infty}$ the supremum norm of $x(\cdot)$.

Let $F:[0,1] \times \mathbb{R}^{n} \leadsto \mathbb{R}^{n}$ be a set-valued map with non-empty compact images and $t_{0} \in[0,1)$. We say that $x(\cdot) \in W^{1,1}\left(\left[t_{0}, 1\right] ; \mathbb{R}^{n}\right)$ is an $F$-trajectory if $x^{\prime}(t) \in F(t, x(t))$ almost everywhere in $\left[t_{0}, 1\right]$ and that it is a feasible $F$ trajectory if in addition it satisfies (1.2). For $\left(t_{0}, x_{0}\right) \in[0,1] \times \mathbb{R}^{n}$, denote by 
$S_{\left[t_{0}, 1\right]}\left(x_{0}\right)$ the set of all $F$-trajectories defined on $\left[t_{0}, 1\right]$ such that $x\left(t_{0}\right)=x_{0}$, by $S_{\left[t_{0}, 1\right]}^{K}\left(x_{0}\right)$ the set of all feasible $F$-trajectories in $S_{\left[t_{0}, 1\right]}\left(x_{0}\right)$ and by $S_{\left[t_{0}, 1\right]}^{r e l}\left(x_{0}\right)$ the set of all solutions to the differential inclusion

$$
\left\{\begin{array}{l}
x^{\prime}(t) \in \operatorname{co} F(t, x(t)) \quad \text { a.e. in }\left[t_{0}, 1\right] \\
x\left(t_{0}\right)=x_{0} .
\end{array}\right.
$$

Let $L_{+}^{1}([0,1])$ be the set of all real valued nonnegative integrable functions defined of $[0,1]$. We impose the following regularity assumptions on $F$

$$
\left\{\begin{array}{l}
F \text { is locally bounded at every }(t, x) \in[0,1] \times \partial K \\
F(\cdot, x) \text { is Lebesgue measurable for every } x \in \mathbb{R}^{n} ; \\
\exists \gamma \in L_{+}^{1}([0,1]), \sup _{v \in F(t, x)}|v| \leq \gamma(t)(1+|x|) \quad \forall(t, x) \in[0,1] \times \mathbb{R}^{n} ; \\
\forall R>0, \exists \lambda_{R} \in L_{+}^{1}([0,1]) \text { such that } \\
F(t, \cdot) \text { is } \lambda_{R}(t)-\text { Lipschitz on } R B \text { for a.e. } t \in[0,1] .
\end{array}\right.
$$

Proposition 2.2. Suppose $(A)$ and that $\forall R>0, \exists \eta, \rho>0$ such that for a.e. $t \in[0,1], \forall y \in(\partial K+\eta B) \cap R B, \forall v \in F(t, y) \backslash \bigcap_{x \in \partial K \cap B(y, \eta)} \operatorname{Int} C_{K}(x), \exists w \in$ $F(t, y)$ satisfying

$$
\max \left\{\langle n, w\rangle,\langle n, w-v\rangle \mid n \in N_{K}^{1}(x), x \in \partial K \cap B(y, \eta)\right\} \leq-\rho .
$$

Then for every $r_{0}>0$ we can find $L>0$ such that $\forall t_{0} \in[0,1), x_{0} \in K \cap$ $r_{0} B, \tilde{x}(\cdot) \in S_{\left[t_{0}, 1\right]}\left(x_{0}\right)$ and $\varepsilon_{0}>0, \exists x(\cdot) \in S_{\left[t_{0}, 1\right]}^{K}\left(x_{0}\right)$ satisfying $x\left(\left(t_{0}, 1\right]\right) \subset$ Int K,

$$
\|x(\cdot)-\tilde{x}(\cdot)\|_{W^{1,1}} \leq L\left(\max _{t \in\left[t_{0}, 1\right]} d_{K}(\tilde{x}(t))+\varepsilon_{0}\right) .
$$

We omit the proof since it follows by exactly the same arguments as those of [16, Proof of Theorem 5]. Let us underline that Theorem 5 in [16] is stated for $F$ upper semicontinuous at every $(t, x) \in[0,1] \times \partial K$ and for a pointwise inward pointing condition imposed for all $(t, x) \in[0,1] \times \partial K$. Then [16, Proposition 7] implies that the assumption as in the above Proposition is satisfied (again for all $t \in[0,1]$ ). Finally, proof of Theorem 5 from [16] remains the same if this assumption holds true only for a.e. $t \in[0,1]$ instead of for all $t \in[0,1]$.

\section{Neighboring feasible trajectories theorems}

Below $\mu^{\ell}$ denotes the Lebesgue measure on $[0,1]$ and Liminf the Kuratowski lower limit. We introduce the following inward pointing condition

$$
\left(\text { IPC) } \left\{\begin{array}{c}
\forall t \in[0,1], \forall x \in \partial K, \exists A_{t, x} \subset[0,1] \text { such that } \mu^{\ell}\left(A_{t, x}\right)=0 \text { and } \\
\forall v \in \operatorname{Limsup}_{(s, y) \rightarrow(t, x)} F(s, y) \text { with } \max _{n \in N_{K}^{1}(x)}\langle n, v\rangle \geq 0, \\
s \notin A_{t, x} \\
\exists w \in \operatorname{Liminf}_{(s, y) \rightarrow(t, x)} F(s, y), \max _{n \in N_{K}^{1}(x)}\langle n, w-v\rangle<0 . \\
s \notin A_{t, x}
\end{array}\right.\right.
$$


Remarks 3.1. $\quad$ i) If $F$ is continuous at every $(t, x) \in[0,1] \times \partial K$, then (IPC) is equivalent to

$$
\left\{\begin{array}{l}
\forall t \in[0,1], x \in \partial K, v \in F(t, x) \text { with } \max _{n \in N_{K}^{1}(x)}\langle n, v\rangle \geq 0, \\
\text { we have } \min _{w \in F(t, x)} \max _{n \in N_{K}^{1}(x)}\langle n, w-v\rangle<0
\end{array}\right.
$$

ii) Condition (IPC) involves normal cones. It has the following equivalent version in terms of interiors of tangent cones:

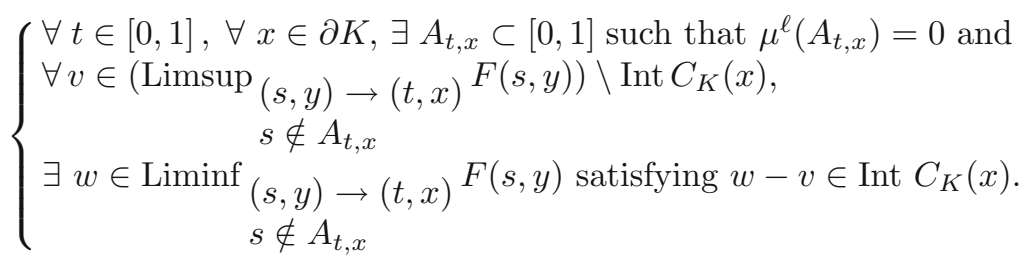

iii) If $\partial K$ is of class $C^{1}$, then the classical inward pointing condition

$$
\min _{w \in F(t, x)}\left\langle n_{x}, w\right\rangle<0 \quad \forall t \in[0,1], \forall x \in \partial K,
$$

where $n_{x}$ denotes the unit outward normal to $K$ at $x$, yields (3.1). The proof of Lemma 3.5 below implies that for a locally bounded $F$, (3.1) is equivalent to the classical inward pointing condition.

iv) The proof of Lemma 3.5 below implies that if (3.1) holds true and $F$ is continuous, then $F(t, x) \cap \operatorname{Int} C_{K}(x) \neq \emptyset$ for all $(t, x) \in[0,1] \times \partial K$. In [4] such condition was used to prove NFT estimates in $\|\cdot\|_{\infty}$ when $F(\cdot, x)$ is left absolutely continuous. To get NFT estimates in $W^{1,1}$ for $F$ merely measurable with respect to time, the stronger (IPC) is crucial.

Theorem 3.2. Assume $(A)$ and $(I P C)$. Then $\forall r_{0}>0, \exists L>0$ such that for all $t_{0} \in[0,1), x_{0} \in K \cap r_{0} B$, and for every $\tilde{x}(\cdot) \in S_{\left[t_{0}, 1\right]}\left(x_{0}\right), \varepsilon_{0}>0$, there exists $x(\cdot) \in S_{\left[t_{0}, 1\right]}^{K}\left(x_{0}\right)$ satisfying $x\left(\left(t_{0}, 1\right]\right) \subset \operatorname{Int} \mathrm{K}$ and

$$
\|x(\cdot)-\tilde{x}(\cdot)\|_{W^{1,1}} \leq L\left(\max _{t \in\left[t_{0}, 1\right]} d_{K}(\tilde{x}(t))+\varepsilon_{0}\right) .
$$

Furthermore, for any feasible $\bar{x}(\cdot) \in S_{\left[t_{0}, 1\right]}^{r e l}\left(x_{0}\right)$ and $\delta>0$ there exists $x(\cdot) \in$ $S_{\left[t_{0}, 1\right]}^{K}\left(x_{0}\right)$ such that $x\left(\left(t_{0}, 1\right]\right) \subset \operatorname{Int} \mathrm{K}$ and $\|x-\bar{x}\|_{\infty}<\delta$.

We will also use the following relaxed inward pointing condition

$$
\left(I P_{r e l}\right)\left\{\begin{aligned}
\forall t \in[0,1], \forall x \in \partial K, \exists A_{t, x} \subset[0,1] \text { such that } \mu^{\ell}\left(A_{t, x}\right)=0 \text { and } \\
\forall v \in \operatorname{Limsup} \\
(s, y) \rightarrow(t, x) F(s, y) \text { with } \max _{n \in N_{K}^{1}(x)}\langle n, v\rangle \geq 0, \\
\quad s \notin A_{t, x} \\
\exists w \in \operatorname{Liminf} \underset{(s, y) \rightarrow(t, x)}{ } \operatorname{co} F(s, y), \max _{n \in N_{K}^{1}(x)}\langle n, w-v\rangle<0 . \\
s \notin A_{t, x}
\end{aligned}\right.
$$

As in Remark $3.1 \mathrm{ii}$ ), the above relaxed condition can be rewritten in the "tangent" form. Theorem 3.3 below is related to Theorem 3.2, however neither one is contained in another. 
Theorem 3.3. Assume $(A),\left(I P_{r e l}\right)$. Then for any $r_{0}>0$ there exist $L, \varepsilon>0$ such that for all $\left(t_{0}, x_{0}\right) \in[0,1] \times\left(K \cap r_{0} B\right)$, for any measurable mapping $[0,1] \ni t \mapsto(\alpha(t), \beta(t)) \in[-\varepsilon, \varepsilon] \times \varepsilon B$, for every solution $\tilde{x}(\cdot)$ of

$$
\left\{\begin{array}{l}
x^{\prime}(t) \in(1+\alpha(t))(\operatorname{co} F(t, x(t))+\beta(t)) \text { for a.e.t } \in\left[t_{0}, 1\right] \\
x\left(t_{0}\right)=x_{0},
\end{array}\right.
$$

and any $\tau_{0}>0$, there exists a trajectory $x(\cdot)$ of $(3.2)$ satisfying $x\left(\left(t_{0}, 1\right]\right) \subset$ Int $\mathrm{K}$ and

$$
\|x(\cdot)-\tilde{x}(\cdot)\|_{W^{1,1}} \leq L\left(\max _{t \in\left[t_{0}, 1\right]} d_{K}(\tilde{x}(t))+\tau_{0}\right) .
$$

Theorem 3.3 and the constructive argument of [4, Proof of Lemma 5.2] imply the following Corollary.

Corollary 3.4. Under assumptions $(A),\left(I P_{\text {rel }}\right)$, for any feasible $x^{\text {rel }}(\cdot) \in$ $S_{\left[t_{0}, 1\right]}^{r e l}\left(x_{0}\right)$ and $\delta>0$ there exists $x(\cdot) \in S_{\left[t_{0}, 1\right]}^{K}\left(x_{0}\right)$ satisfying $x\left(\left(t_{0}, 1\right]\right) \subset$ Int $\mathrm{K}$ and $\max _{t \in\left[t_{0}, 1\right]}\left|x(t)-x^{\mathrm{rel}}(t)\right| \leq \delta$.

To prove Theorem 3.2 we need the following lemma.

Lemma 3.5. Assume $(I P C)$ and that $F$ is locally bounded at every $(t, x) \in$ $[0,1] \times \partial K$. Then $\forall R>0$ there exist $\eta, \rho>0$ and $A \subset[0,1]$ of $\mu^{\ell}(A)=0$ such that $\forall t \in[0,1] \backslash A, \forall y \in(\partial K+\eta B) \cap R B$, $\forall v \in F(t, y) \backslash \bigcap_{x \in \partial K \cap B(y, \eta)} \operatorname{Int} C_{K}(x), \exists w \in F(t, y)$ satisfying

$$
\max \left\{\langle n, w\rangle,\langle n, w-v\rangle \mid n \in N_{K}^{1}(x), x \in \partial K \cap B(y, \eta)\right\} \leq-\rho .
$$

Remark 3.6. By the above lemma, the local boundedness of $F$ on $[0,1] \times \partial K$ and assumption (IPC) imply that Int $C_{K}(x) \neq \emptyset$ for all $x \in \partial K$.

Proof. Fix $R>0$ and $(\bar{t}, \bar{x}) \in[0,1] \times(\partial K \cap 2 R B)$. In the following we will write

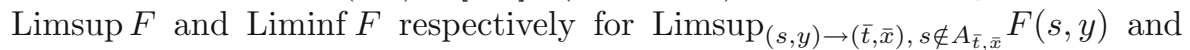

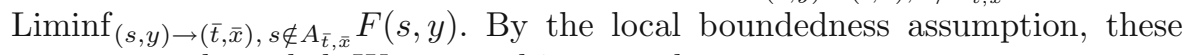
two sets are bounded. We proceed in several steps.

Step 1. We claim $\exists \gamma_{\bar{t}, \bar{x}}>0$ such that $\forall v \in \operatorname{Limsup} F \backslash \operatorname{Int} C_{K}(\bar{x}), \exists w \in$ Liminf $F$ satisfying $\max _{n \in N_{K}^{1}(\bar{x})}\langle n, w-v\rangle \leq-\gamma_{\bar{t}, \bar{x}}$.

Indeed fix $\bar{v} \in \operatorname{Limsup} F \backslash \operatorname{Int} C_{K}(\bar{x}), \gamma_{\bar{v}}>0$ and $w \in \operatorname{Liminf} F$ such that $\max _{n \in N_{K}^{1}(\bar{x})}\langle n, w-\bar{v}\rangle \leq-2 \gamma_{\bar{v}}$. Then for all $v \in B\left(\bar{v}, \gamma_{\bar{v}}\right)$ we have $\max _{n \in N_{K}^{1}(\bar{x})}\langle n, w-v\rangle \leq-\gamma_{\bar{v}}$. The claim follows from the compactness of the set Limsup $F \backslash \operatorname{Int} C_{K}(\bar{x})$.

Step 2. We claim that for every $v \in \operatorname{Limsup} F \backslash \operatorname{Int} C_{K}(\bar{x})$, we may choose $w$ as in Step 1 such that $w \in \operatorname{Int} C_{K}(\bar{x})$.

Suppose by contradiction that for some $v \in \operatorname{Limsup} F \backslash \operatorname{Int} C_{K}(\bar{x})$, there is no $w \in \operatorname{Liminf} F \cap \operatorname{Int} C_{K}(\bar{x})$ satisfying $\max _{n \in N_{K}^{1}(\bar{x})}\langle n, w-v\rangle \leq-\gamma_{\bar{t}, \bar{x}}$. Let $w_{1}$ be as in Step 1 corresponding to this $v$. Since $w_{1} \notin \operatorname{Int} C_{K}(\bar{x})$ and Liminf $F \subset$ Limsup $F$, by Step 1, there exists $w_{2} \in \operatorname{Liminf} F$ satisfying $\max _{n \in N_{K}^{1}(\bar{x})}\left\langle n, w_{2}-\right.$ $\left.w_{1}\right\rangle \leq-\gamma_{\bar{t}, \bar{x}}$. Then $\max _{n \in N_{K}^{1}(\bar{x})}\left\langle n, w_{2}-v\right\rangle \leq-2 \gamma_{\bar{t}, \bar{x}}$ and $w_{2} \notin \operatorname{Int} C_{K}(\bar{x})$. Using the induction argument we construct a sequence $w_{i} \in \operatorname{Liminf} F$ such that, for every $i, \max _{n \in N_{K}^{1}(\bar{x})}\left\langle n, w_{i}-v\right\rangle \leq-i \gamma_{\bar{t}, \bar{x}}$. Since Liminf $F$ is bounded, this inequality leads to a contradiction. 
Step 3. Arguments analogous to those of Step 1 imply the existence of $\rho_{\bar{t}, \bar{x}}>0$ such that $\forall v \in \operatorname{Limsup} F \backslash \operatorname{Int} C_{K}(\bar{x}), \exists w \in \operatorname{Liminf} F$ satisfying

$$
\max _{n \in N_{K}^{1}(\bar{x})}\{\langle n, w\rangle,\langle n, w-v\rangle\} \leq-2 \rho_{\bar{t}, \bar{x}} .
$$

Step 4. We claim that there exists $\eta_{\bar{t}, \bar{x}}>0$ such that for all $(t, y) \in B\left(\bar{t}, \eta_{\bar{t}, \bar{x}}\right) \times$ $\left(\left(\partial K \cap B\left(\bar{x}, \eta_{\bar{t}, \bar{x}}\right)\right)+\eta_{\bar{t}, \bar{x}} B\right)$ with $t \in[0,1] \backslash A_{\bar{t}, \bar{x}}, \forall v \in F(t, y) \backslash \bigcap_{x \in \partial K \cap B\left(y, \eta_{\bar{t}, \bar{x}}\right)}$ Int $C_{K}(x), \exists w \in F(t, y)$ satisfying

$$
\max \left\{\langle n, w\rangle,\langle n, w-v\rangle \mid n \in N_{K}^{1}(x), x \in \partial K \cap B\left(y, \eta_{\bar{t}, \bar{x}}\right)\right\} \leq-\rho_{\bar{t}, \bar{x}} .
$$

Suppose by contradiction that there exist $\left(t_{i}, y_{i}\right) \rightarrow(\bar{t}, \bar{x}), t_{i} \in[0,1] \backslash A_{\bar{t}, \bar{x}}$, $v_{i} \in F\left(t_{i}, y_{i}\right), x_{i} \rightarrow^{\partial K} \bar{x}$ and $n_{i} \in N_{K}^{1}\left(x_{i}\right)$ such that $\left\langle n_{i}, v_{i}\right\rangle \geq 0$ and $\forall w_{i} \in$ $F\left(t_{i}, y_{i}\right)$ we can find $x_{i}^{\prime} \rightarrow^{\partial K} \bar{x}, n_{i}^{\prime} \in N_{K}^{1}\left(x_{i}^{\prime}\right)$ satisfying

$$
\left\langle n_{i}^{\prime}, w_{i}\right\rangle \vee\left\langle n_{i}^{\prime}, w_{i}-v_{i}\right\rangle>-\rho_{\bar{t}, \bar{x}}
$$

Taking subsequences we may assume that $\lim _{i \rightarrow \infty} v_{i}=v \in \operatorname{Limsup} F$ and $\lim _{i \rightarrow \infty} n_{i}=n, \lim _{i \rightarrow \infty} n_{i}^{\prime}=n^{\prime}$. Since the map $x \leadsto N_{K}^{1}(x)$ is upper semicontinuous, we have $n, n^{\prime} \in N_{K}^{1}(\bar{x})$ and $\langle n, v\rangle \geq 0$. Then $v \notin \operatorname{Int} C_{K}(\bar{x})$. Consider $w \in \operatorname{Liminf} F$ as in Step 3 corresponding to this $v$ and let $w_{i} \in F\left(t_{i}, y_{i}\right)$ be such that $\lim _{i \rightarrow \infty} w_{i}=w$. From (3.3) we deduce that $\left\langle n^{\prime}, w\right\rangle \vee\left\langle n^{\prime}, w-v\right\rangle \geq-\rho_{\bar{t}, \bar{x}}$, contradicting the choice of $w$.

Step 5. Consider a finite subcovering $\left\{\stackrel{\circ}{B}\left(\left(t_{k}, x_{k}\right), \eta_{t_{k}, x_{k}}\right)\right\}_{k=1, \ldots, N}$ of $[0,1] \times$ $(\partial K \cap 2 R B)$. The assertions of the Lemma are satisfied if we set $A=\bigcup_{k} A_{t_{k}, x_{k}}$, $\eta=\min _{k}\left\{R, \eta_{t_{k}, x_{k}}\right\}$ and $\rho=\min _{k} \rho_{t_{k}, x_{k}}$.

Proof of Theorem 3.2. From Lemma 3.5 and Proposition 2.2 we get the first statement. Fix a feasible $\bar{x}(\cdot) \in S_{\left[t_{0}, 1\right]}^{r e l}\left(x_{0}\right), \delta>0$ and set $r_{0}=\left|x_{0}\right|$. Let $L>0$ be as in the first claim of the theorem. By the relaxation theorem, for some $\tilde{x}(\cdot) \in S_{\left[t_{0}, 1\right]}\left(x_{0}\right),\|\tilde{x}(\cdot)-\bar{x}(\cdot)\|_{\infty}<\frac{\delta}{3(L+1)}$. Thus $\max _{t \in\left[t_{0}, 1\right]} d_{K}(\tilde{x}(t))<$ $\frac{\delta}{3 L}$. Set $\varepsilon_{0}=\frac{\delta}{3 L}$. Then for some $x(\cdot) \in S_{\left[t_{0}, 1\right]}^{K}\left(x_{0}\right)$ we have $x\left(\left(t_{0}, 1\right]\right) \subset$ Int $\mathrm{K},\|\tilde{\mathrm{x}}(\cdot)-\mathrm{x}(\cdot)\|_{\infty}<\frac{2 \delta}{3}$. Thus $\|\bar{x}(\cdot)-x(\cdot)\|_{\infty}<\delta$.

To prove Theorem 3.3 we need the following lemma.

Lemma 3.7. Assume $\left(I P_{r e l}\right)$ and that $F$ is locally bounded at every $(t, x) \in$ $[0,1] \times \partial K$. Then for every $R>0$ there exist $\eta, \rho, \varepsilon>0$ and $A \subset[0,1]$ of $\mu^{\ell}(A)=0$ such that for all $t \in[0,1] \backslash A, y \in(\partial K+\eta B) \cap R B, \alpha \in[-\varepsilon, \varepsilon]$, $\beta \in \varepsilon B$ and every

$$
v \in(1+\alpha)(\operatorname{co} F(t, y)+\beta) \backslash \bigcap_{x \in \partial K \cap B(y, \eta)} \operatorname{Int} C_{K}(x)
$$

we can find $w \in(1+\alpha)(\operatorname{co} F(t, y)+\beta)$ satisfying

$$
\max \left\{\langle n, w\rangle,\langle n, w-v\rangle \mid n \in N_{K}^{1}(x), x \in \partial K \cap B(y, \eta)\right\} \leq-\rho .
$$

Proof. Fix $R>0$ and $(\bar{t}, \bar{x}) \in[0,1] \times(\partial K \cap 2 R B)$. Define Limsup co $F$ and Liminf $c o F$ as in the proof of Lemma 3.5 with $F$ replaced by co $F$. Observe 
that, as in Steps 1-3 of the proof of Lemma 3.5, there exists $\gamma_{\bar{t}, \bar{x}}>0$ such that $\forall v \in \operatorname{Limsup} F \backslash \operatorname{Int} C_{K}(\bar{x}), \exists w \in \operatorname{Liminf} c o F$ satisfying

$$
\max _{n \in N_{K}^{1}(\bar{x})}\{\langle n, w\rangle,\langle n, w-v\rangle\} \leq-\gamma_{\bar{t}, \bar{x}} .
$$

(a) We claim that for every $v \in(\operatorname{coLimsup} F) \backslash \operatorname{Int} C_{K}(\bar{x})$ there exist $\lambda^{1}>0, \lambda^{2} \geq 0, v^{1} \in \operatorname{co}\left(\operatorname{Limsup} F \backslash \operatorname{Int} C_{K}(\bar{x})\right), v^{2} \in \mathbb{R}^{n}$ and $w^{1} \in \operatorname{Liminf}$ co $F$ such that $\lambda^{1}+\lambda^{2}=1, v=\lambda^{1} v^{1}+\lambda^{2} v^{2}$, where $v^{2}=0$ if $\lambda^{2}=0$ and $v^{2} \in$ co (Limsup $\left.F \cap \operatorname{Int} C_{K}(\bar{x})\right)$ otherwise, and

$$
\max _{n \in N_{K}^{1}(\bar{x})}\left\{\left\langle n, w^{1}\right\rangle,\left\langle n, w^{1}-v^{1}\right\rangle\right\} \leq-\gamma_{\bar{t}, \bar{x}} .
$$

Indeed, for $v \in(\operatorname{coLimsup} F) \backslash \operatorname{Int} C_{K}(\bar{x})$, let $\lambda_{\alpha}>0, v_{\alpha} \in \operatorname{Limsup} F, \alpha=$ $1, \ldots, m \leq n+1$, be such that $\sum_{\alpha=1}^{m} \lambda_{\alpha}=1$ and $\sum_{\alpha=1}^{m} \lambda_{\alpha} v_{\alpha}=v$. By reordering we may assume that $v_{1}, \ldots, v_{l} \notin \operatorname{Int} C_{K}(\bar{x})$ and $v_{l+1}, \ldots, v_{m} \in \operatorname{Int} C_{K}(\bar{x})$. Observe that $l \geq 1$, since otherwise $v \in \operatorname{Int} C_{K}(\bar{x})$. Define $\lambda^{1}=\sum_{\alpha=1}^{l} \lambda_{\alpha}$, $\lambda^{2}=1-\lambda^{1}, v^{1}=\frac{1}{\lambda^{1}} \sum_{\alpha=1}^{l} \lambda_{\alpha} v_{\alpha}, v^{2}=\frac{1}{\lambda^{2}} \sum_{\alpha=l+1}^{m} \lambda_{\alpha} v_{\alpha}$ if $l<m$ and $v^{2}=0$ if $l=m$. Then $v=\lambda^{1} v^{1}+\lambda^{2} v^{2}$ and $v^{1} \notin \operatorname{Int} C_{K}(\bar{x})$, since otherwise we would get $v \in \operatorname{Int} C_{K}(\bar{x})$. For each $\alpha=1, \ldots, l$, let $w_{\alpha} \in \operatorname{Liminf} c o F$ be such that $\max _{n \in N_{K}^{1}(\bar{x})}\left\{\left\langle n, w_{\alpha}\right\rangle,\left\langle n, w_{\alpha}-v_{\alpha}\right\rangle\right\} \leq-\gamma_{\bar{t}, \bar{x}}$. Then the vector $w^{1}=$ $\frac{1}{\lambda^{1}} \sum_{\alpha=1}^{l} \lambda_{\alpha} w_{\alpha}$ is as required.

(b) We claim $\exists \rho_{\bar{t}, \bar{x}}>0$ such that for all $v \in(\operatorname{coLimsup} F) \backslash \operatorname{Int} C_{K}(\bar{x})$ we can find $w \in$ Liminf $c o F$ satisfying $\max _{n \in N_{K}^{1}(\bar{x})}\{\langle n, w\rangle,\langle n, w-v\rangle\} \leq-2 \rho_{\bar{t}, \bar{x}}$.

Indeed suppose for a moment that for some $v_{i} \in(c o \operatorname{Limsup} F) \backslash \operatorname{Int} C_{K}(\bar{x})$ and for any choice of $w \in \operatorname{Liminf} \operatorname{co} F$ we have

$$
\max _{n \in N_{K}^{1}(\bar{x})}\left\{\langle n, w\rangle,\left\langle n, w-v_{i}\right\rangle\right\}>-\frac{1}{i} .
$$

For each $i$ let $\lambda_{i}^{1}>0, \lambda_{i}^{2} \geq 0, \lambda_{i}^{1}+\lambda_{i}^{2}=1, v_{i}^{1} \in \operatorname{co}\left(\operatorname{Limsup} F \backslash \operatorname{Int} C_{K}(\bar{x})\right), v_{i}^{2} \in$ $\mathbb{R}^{n}$ be such that $v_{i}^{2} \in$ co $\left(\operatorname{Limsup} F \cap \operatorname{Int} C_{K}(\bar{x})\right)$ if $\lambda_{i}^{2}>0$ and $v_{i}^{2}=0$ otherwise, $v_{i}=\lambda_{i}^{1} v_{i}^{1}+\lambda_{i}^{2} v_{i}^{2}$ and let $w_{i}^{1} \in \operatorname{Liminf} \operatorname{co} F$ satisfy

$$
\max _{n \in N_{K}^{1}(\bar{x})}\left\{\left\langle n, w_{i}^{1}\right\rangle,\left\langle n, w_{i}^{1}-v_{i}^{1}\right\rangle\right\} \leq-\gamma_{\bar{t}, \bar{x}} .
$$

Taking subsequences we may assume that $\lambda_{i}^{1} \rightarrow \lambda^{1}, \lambda_{i}^{2} \rightarrow \lambda^{2}, \lambda^{1}+\lambda^{2}=1, v_{i}^{1} \rightarrow$ $v^{1} \in$ co $\left(\operatorname{Limsup} F \backslash \operatorname{Int} C_{K}(\bar{x})\right), v_{i}^{2} \rightarrow v^{2} \in \mathbb{R}^{n}$ and $w_{i}^{1} \rightarrow w^{1} \in \operatorname{Liminf}$ co $F$. Then $v^{2} \in$ co $\left(\operatorname{Limsup} F \cap C_{K}(\bar{x})\right)$ whenever $\lambda^{2}>0$. Clearly $\lim _{i \rightarrow \infty} v_{i}=$ $v:=\lambda^{1} v^{1}+\lambda^{2} v^{2}, v \in(\operatorname{coLimsup} F) \backslash \operatorname{Int} C_{K}(\bar{x})$ and

$$
\max _{n \in N_{K}^{1}(\bar{x})}\left\{\left\langle n, w^{1}\right\rangle,\left\langle n, w^{1}-v^{1}\right\rangle\right\} \leq-\gamma_{\bar{t}, \bar{x}} .
$$

Moreover, for all $w \in \operatorname{Liminf} c o F$,

$$
\max _{n \in N_{K}^{1}(\bar{x})}\{\langle n, w\rangle,\langle n, w-v\rangle\} \geq 0
$$

If $\lambda^{2}=0$, then the last two inequalities are in contradiction. Hence $\lambda^{2}>0$.

Suppose first that $v^{2} \in \partial C_{K}(\bar{x})$. Then for some $\mu_{j}>0$ and for some $\tilde{v}_{j} \in \operatorname{Limsup} F \cap C_{K}(\bar{x})$, we have $\sum_{j=0}^{m} \mu_{j}=1, m \leq n$ and $v^{2}=\sum_{j=0}^{m} \mu_{j} \tilde{v}_{j}$. 
Since $C_{K}(\bar{x})$ is convex, $\tilde{v}_{j} \in \partial C_{K}(\bar{x})$ for every $j$. By the remark at the beginning of the proof, $\max _{n \in N_{K}^{1}(\bar{x})}\left\{\left\langle n, w_{j}\right\rangle,\left\langle n, w_{j}-\tilde{v}_{j}\right\rangle\right\} \leq-\gamma_{\bar{t}, \bar{x}}$ for some $w_{j} \in$ Liminf $c o F$ and each $j$. Set $w^{2}=\sum_{j=0}^{m} \mu_{j} w_{j}$. Then $w^{2} \in \operatorname{Liminf} c o F$ and $\max _{n \in N_{K}^{1}(\bar{x})}\left\{\left\langle n, w^{2}\right\rangle,\left\langle n, w^{2}-v^{2}\right\rangle\right\} \leq-\gamma_{\bar{t}, \bar{x}}$. Setting $w=\lambda^{1} w^{1}+\lambda^{2} w^{2}$, we obtain a contradiction with (3.4).

On the other hand, if $v^{2} \in \operatorname{Int} C_{K}(\bar{x})$, then $\lambda^{1}>0$. Setting $w=\lambda^{1} w^{1}+$ $\lambda^{2} v^{2}$, we obtain $\max _{n \in N_{K}^{1}(\bar{x})}\{\langle n, w\rangle,\langle n, w-v\rangle\} \leq-\lambda^{1} \gamma_{\bar{t}, \bar{x}}<0$. This contradicts (3.4) and proves our claim.

(c) We claim that for some positive $\eta_{\bar{t}, \bar{x}}, \varepsilon_{\bar{t}, \bar{x}}$ and for all $\alpha \in$ $\left[-\varepsilon_{\bar{t}, \bar{x}}, \varepsilon_{\bar{t}, \bar{x}}\right], \beta \in \varepsilon_{\bar{t}, \bar{x}} B,(t, y) \in B\left(\bar{t}, \eta_{\bar{t}, \bar{x}}\right) \times\left(\left(\partial K \cap B\left(\bar{x}, \eta_{\bar{t}, \bar{x}}\right)\right)+\eta_{\bar{t}, \bar{x}} B\right)$ with $t \in[0,1] \backslash A_{\bar{t}, \bar{x}}$ and every

$$
v \in(1+\alpha)(\operatorname{coF}(t, y)+\beta) \backslash \bigcap_{x \in \partial K \cap B\left(y, \eta_{\bar{t}, \bar{x}}\right)} \operatorname{Int} C_{K}(x),
$$

there exists $w \in(1+\alpha)(\operatorname{co} F(t, y)+\beta)$ such that

$$
\max \left\{\langle n, w\rangle,\langle n, w-v\rangle \mid n \in N_{K}^{1}(x), x \in \partial K \cap B\left(y, \eta_{\bar{t}, \bar{x}}\right)\right\} \leq-\rho_{\bar{t}, \bar{x}} .
$$

We argue by contradiction as in Step 4 of the proof of Lemma 3.5. Suppose that there exist $\left(t_{i}, y_{i}\right) \rightarrow(\bar{t}, \bar{x}), t_{i} \in[0,1] \backslash A_{\bar{t}, \bar{x}},\left(\alpha_{i}, \beta_{i}\right) \rightarrow(0,0)$ and

$$
\bar{v}_{i} \in\left(1+\alpha_{i}\right)\left(\operatorname{coF}\left(t_{i}, y_{i}\right)+\beta_{i}\right) \backslash \bigcap_{x \in \partial K \cap B\left(y_{i}, \frac{1}{i}\right)} \operatorname{Int} C_{K}(x)
$$

such that for every $\bar{w}_{i} \in\left(1+\alpha_{i}\right)\left(\operatorname{co} F\left(t_{i}, y_{i}\right)+\beta_{i}\right)$ we can find $x_{i} \rightarrow^{\partial K} \bar{x}, n_{i} \in$ $N_{K}^{1}\left(x_{i}\right)$ satisfying

$$
\left\langle n_{i}, \bar{w}_{i}\right\rangle \vee\left\langle n_{i}, \bar{w}_{i}-\bar{v}_{i}\right\rangle>-\rho_{\bar{t}, \bar{x}}
$$

Observe that if $v$ is a cluster point of $\bar{v}_{i}$, then $v \notin \operatorname{Int} C_{K}(\bar{x})$. Moreover, $v \in \operatorname{Limsup}_{(s, y) \rightarrow(\bar{t}, \bar{x}), s \notin A_{\bar{t}, \bar{x}},(\alpha, \beta) \rightarrow(0,0)}(1+\alpha)(\operatorname{co} F(s, y)+\beta) \subset \operatorname{coLimsup} F$.

Hence, by (b), there exists $w \in \operatorname{Liminf} c o F$ satisfying

$$
\max _{n \in N_{K}^{1}(\bar{x})}\{\langle n, w\rangle,\langle n, w-v\rangle\} \leq-2 \rho_{\bar{t}, \bar{x}} .
$$

Let $w_{i} \in \operatorname{co} F\left(t_{i}, x_{i}\right)$ be such that $\lim _{i \rightarrow \infty} w_{i}=w$ and $\bar{w}_{i}:=\left(1+\alpha_{i}\right)\left(w_{i}+\beta_{i}\right)$. If $n \in N_{K}^{1}(\bar{x})$ is a cluster point of $n_{i}$, then from (3.5) and (3.6) we deduce $-\rho_{\bar{t}, \bar{x}} \leq\langle n, w\rangle \vee\langle n, w-v\rangle \leq-2 \rho_{\bar{t}, \bar{x}}$. The obtained contradiction proves our claim.

The statement of the Lemma follows from (c) if we define $\eta, \rho$ and $A$ as in Step 5 of the proof of Lemma 3.5 and $\varepsilon=\min _{k} \varepsilon_{t_{k}, x_{k}}$.

Proof of Theorem 3.3. In the light of Lemma 3.7, Theorem 3.3 follows from Proposition 2.2 applied with $F(t, x)$ replaced by $(1+\alpha(t))(\operatorname{co} F(t, x)+\beta(t))$. 


\section{Variational inclusions}

Recall that the adjacent tangent cone to $K$ at $x \in K$ is defined by

$$
I_{K}(x)=\left\{v \in \mathbb{R}^{n} \mid \lim _{h \rightarrow 0+} \frac{d_{K}(x+h v)}{h}=0\right\} .
$$

Let $F(t, \cdot)$ be locally Lipschitz. In this section, for every $y \in F(t, x)$ we denote by $d_{x} F(t, x, y)$ the adjacent derivative of $F(t, \cdot)$ at $(x, y)$, that is the set-valued map from $\mathbb{R}^{n}$ into $\mathbb{R}^{n}$ defined by

$$
d_{x} F(t, x, y)(u)=\left\{v \in \mathbb{R}^{n} \mid \lim _{h \rightarrow 0+} \operatorname{dist}\left(v, \frac{F(t, x+h u)-y}{h}\right)=0\right\} .
$$

The two theorems below generalize variational inclusions from [14,22].

Theorem 4.1. Assume $(A),(I P C)$ and fix $x(\cdot) \in S_{[0,1]}^{K}\left(x_{0}\right)$. Let $h_{i} \rightarrow 0+, w_{i} \rightarrow$ $w_{0} \in I_{K}\left(x_{0}\right)$ be such that $x_{0}+h_{i} w_{i} \in K$ for all $i$ and consider a trajectory $w(\cdot)$ of the differential inclusion

$$
\begin{cases}w^{\prime}(t) \in d_{x} F\left(t, x(t), x^{\prime}(t)\right)(w(t)) & \text { a.e. in }[0,1] \\ w(t) \in I_{K}(x(t)) & \text { for all } t \in[0,1] \\ w(0)=w_{0} & \end{cases}
$$

If

$$
\lim _{h \rightarrow 0+} \frac{1}{h} \max _{t \in[0,1]} d_{K}(x(t)+h w(t))=0,
$$

then there exists a sequence of trajectories $x_{i}(\cdot) \in S_{[0,1]}^{K}\left(x_{0}+h_{i} w_{i}\right)$ such that $\frac{x_{i}(\cdot)-x(\cdot)}{h_{i}}$ converge in $W^{1,1}$ to $w(\cdot)$.

Remark 4.2. Assumption (4.1) is satisfied for instance if for every $R>0$ there exists $\phi: \mathbb{R}_{+} \rightarrow \mathbb{R}_{+}$such that $\lim _{h \rightarrow 0+} \frac{\phi(h)}{h}=0$ and $d_{K}(x+h v) \leq \phi(h)$ for all $x \in \partial K \cap R B$ and $v \in I_{K}(x) \cap S^{n-1}$. In particular, if $K$ is sleek (in the sense that for all $x \in \partial K, C_{K}(x)$ is equal to the contingent cone to $K$ at $x$, cf. [2]), then (4.1) is satisfied.

Note that by the very definition of the tangent cone $I_{K}(x(t))$, for all $t \in[0,1]$

$$
\lim _{h \rightarrow 0+} \frac{d_{K}(x(t)+h w(t))}{h}=0 .
$$

However, in general, the above convergence is not uniform with respect to $t$.

Proof. Set $y_{i}(t)=x(t)+h_{i}\left(w(t)+w_{i}-w_{0}\right)$ and $p_{i}(t)=\operatorname{dist}\left(y_{i}^{\prime}(t), F\left(t, y_{i}(t)\right)\right)$. Since $w^{\prime}(t) \in d_{x} F\left(t, x(t), x^{\prime}(t)\right)(w(t))$ a.e., from the local Lipschitz continuity of $F(t, \cdot)$ we get $\int_{0}^{1} p_{i}(t) d t=o\left(h_{i}\right)$. This and Filippov's theorem [13] yield the existence of $\tilde{x}_{i}(\cdot) \in S_{[0,1]}\left(x_{0}+h_{i} w_{i}\right)$ such that $\frac{\tilde{x}_{i}(\cdot)-y_{i}(\cdot)}{h_{i}} \rightarrow W^{1,1} 0$. From the definition of $y_{i}(\cdot)$ it follows that $\frac{\tilde{x}_{i}(\cdot)-x(\cdot)}{h_{i}} \rightarrow W^{1,1} w(\cdot)$. By Theorem 3.2 there exist $L>0$ and $x_{i}(\cdot) \in S_{[0,1]}^{K}\left(x_{0}+h_{i} w_{i}\right)$ satisfying

$$
\left\|x_{i}(\cdot)-\tilde{x}_{i}(\cdot)\right\|_{W^{1,1}} \leq 2 L \max _{t \in[0,1]} d_{K}\left(\tilde{x}_{i}(t)\right) .
$$


Observe next that

$$
\begin{aligned}
d_{K}\left(\tilde{x}_{i}(t)\right) & \leq\left|\tilde{x}_{i}(t)-y_{i}(t)\right|+d_{K}\left(x(t)+h_{i}\left(w(t)+w_{i}-w_{0}\right)\right) \\
& \leq\left|\tilde{x}_{i}(t)-y_{i}(t)\right|+h_{i}\left|w_{i}-w_{0}\right|+d_{K}\left(x(t)+h_{i} w(t)\right) .
\end{aligned}
$$

By assumption (4.1), when $i \rightarrow \infty, \frac{1}{h_{i}} d_{K}\left(x(\cdot)+h_{i} w(\cdot)\right)$ converg uniformly to 0 . Consequently, $\frac{1}{h_{i}}\left\|\tilde{x}_{i}(\cdot)-x_{i}(\cdot)\right\|_{W^{1,1}} \rightarrow 0$ and $\frac{x_{i}(\cdot)-x(\cdot)}{h_{i}}$ converge to $w(\cdot)$ in $W^{1,1}$ when $i \rightarrow \infty$.

Theorem 4.3. Assume $(A)$ and $\left(I P_{\text {rel }}\right)$. Let $x(\cdot), h_{i}, w_{i}$ and $w_{0}$ be as in Theorem 4.1. If $w(\cdot)$ is a solution of the differential inclusion

$$
\begin{cases}w^{\prime}(t) \in d_{x} \operatorname{co} F\left(t, x(t), x^{\prime}(t)\right)(w(t)) & \text { a.e. in }[0,1] \\ w(t) \in I_{K}(x(t)) & \text { for all } t \in[0,1] \\ w(0)=w_{0}, & \end{cases}
$$

and (4.1) holds true, then there exists a sequence of feasible trajectories $x_{i}(\cdot) \in$ $S_{[0,1]}^{K}\left(x_{0}+h_{i} w_{i}\right)$ such that $\frac{x_{i}(\cdot)-x(\cdot)}{h_{i}}$ converges uniformly to $w(\cdot)$.

Proof. By replacing $F$ by co $F$ in the proof of Theorem 4.1, we show that there exist $\tilde{x}_{i} \in S_{[0,1]}^{r e l}\left(x_{0}+h_{i} w_{i}\right)$ such that $\frac{\tilde{x}_{i}(\cdot)-x(\cdot)}{h_{i}}$ converge uniformly to $w(\cdot)$. By Theorem 3.3 there exist $L>0$ and $x_{i}^{r e l}(\cdot) \in S_{[0,1]}^{r e l}\left(x_{0}+h_{i} w_{i}\right)$ satisfying $x_{i}^{r e l}([0,1]) \subset K$ and

$$
\left\|x_{i}^{r e l}(\cdot)-\tilde{x}_{i}(\cdot)\right\|_{W^{1,1}} \leq L\left(\max _{t \in[0,1]} d_{K}\left(\tilde{x}_{i}(t)\right)+\frac{h_{i}}{i}\right) .
$$

From assumption (4.1) we deduce that $\frac{x_{i}^{r e l}(\cdot)-\tilde{x}_{i}(\cdot)}{h_{i}}$ converge uniformly to zero when $i \rightarrow \infty$. By Corollary 3.4, there exist $x_{i}(\cdot) \in S_{[0,1]}^{K}\left(x_{0}+h_{i} w_{i}\right)$ such that $\left\|x_{i}(\cdot)-x_{i}^{r e l}(\cdot)\right\|_{L^{\infty}}=o\left(h_{i}\right)$. Thus $\frac{x_{i}(\cdot)-x(\cdot)}{h_{i}}$ converge uniformly to $w(\cdot)$ when $i \rightarrow \infty$.

\section{Sensitivity interpretation of the adjoint state}

Let $g(\cdot): \mathbb{R}^{n} \rightarrow \mathbb{R}, x_{0} \in K$ and consider the following Mayer problem

$$
\min \left\{g(x(1)) \mid x(\cdot) \in S_{[0,1]}^{K}\left(x_{0}\right)\right\} .
$$

The relaxed Mayer problem is the same one, but with differential inclusion (1.1) replaced by the relaxed inclusion (2.1) for $t_{0}=0$. The value function $V:[0,1] \times \mathbb{R}^{n} \rightarrow \mathbb{R} \cup\{+\infty\}$ is defined by (1.4).

Theorem 5.1. Assume $(A)$, $\left(I P_{\text {rel }}\right)$, that $g$ is locally Lipschitz and that $\gamma$ is essentially bounded. Then $V$ is locally Lipschitz on $[0,1] \times K$ and is equal to the value function of the relaxed Mayer problem. Furthermore if $\bar{x}(\cdot)$ is an optimal solution to the Mayer problem, then it is also optimal for the relaxed Mayer problem.

Remark 5.2. The essential boundedness of $\gamma$ is needed to have local Lipschitz continuity of $V$ with respect to the time variable. 
Proof. Denote by $V^{\text {rel }}$ the value function of the relaxed Mayer problem. Fix $\left(t_{0}, y_{0}\right) \in[0,1] \times K$. Clearly $V^{r e l}\left(t_{0}, y_{0}\right) \leq V\left(t_{0}, y_{0}\right)$. Fix $\varepsilon>0$ and set $r=\left|y_{0}\right|$, $\Gamma=\|\gamma(\cdot)\|_{\infty}$. Let $\lambda_{1}>0$ be a Lipschitz constant of $g$ on $B\left(0,(1+r) e^{\Gamma}\right)$.

Consider a feasible $x^{r e l}(\cdot) \in S_{\left[t_{0}, 1\right]}^{r e l}\left(y_{0}\right)$ satisfying $V^{r e l}\left(t_{0}, y_{0}\right)=g\left(x^{r e l}(1)\right)$. By Corollary 3.4 there exists $x(\cdot) \in S_{\left[t_{0}, 1\right]}^{K}\left(y_{0}\right),\left\|x(\cdot)-x^{r e l}(\cdot)\right\|_{\infty} \leq \frac{\varepsilon}{\lambda_{1}}$. Then

$$
V\left(t_{0}, y_{0}\right) \leq g(x(1)) \leq g\left(x^{r e l}(1)\right)+\lambda_{1}\left|x(1)-x^{r e l}(1)\right| \leq V^{r e l}\left(t_{0}, y_{0}\right)+\varepsilon
$$

By the arbitrariness of $\varepsilon, V\left(t_{0}, y_{0}\right)=V^{r e l}\left(t_{0}, y_{0}\right)$. Thus any optimal solution of the Mayer problem is also optimal for the relaxed Mayer problem.

To check the local Lipschitz continuity of $V$ on $[0,1] \times K$ it is sufficient to prove that for any compact subset $Q$ of $K$ there exist positive constants $L_{1}$ and $L_{2}$ such that $V(t, \cdot)$ is $L_{1}$-Lipschitz continuous on $Q$ for all $t \in[0,1]$ and $V\left(\cdot, y_{0}\right)$ is $L_{2}$-Lipschitz continuous on $[0,1]$ for all $y_{0} \in Q$. Fix such $Q$ and consider $t \in[0,1], y_{1}, y_{2} \in Q, y_{1} \neq y_{2}$. Assuming $Q \subset R B$, let $\lambda_{2}$ be a Lipschitz constant of $g$ on $B\left(0,(1+R) e^{\Gamma}\right)$. Let $x_{1}(\cdot) \in S_{[t, 1]}^{K}\left(y_{1}\right)$ satisfy $g\left(x_{1}(1)\right) \leq V\left(t, y_{1}\right)+\left|y_{1}-y_{2}\right|$. By Theorem 3.3 and Filippov's theorem [1,13], there exists a constant $M$ depending only on $R$ such that for some $x_{2}(\cdot) \in S_{[t, 1]}^{K}\left(y_{2}\right)$ we have $\left\|x_{1}(\cdot)-x_{2}(\cdot)\right\|_{\infty} \leq M\left|y_{1}-y_{2}\right|$. Then for $L_{1}:=\lambda_{2} M+1$,

$$
V\left(t, y_{2}\right) \leq g\left(x_{2}(1)\right) \leq g\left(x_{1}(1)\right)+\lambda_{2} M\left|y_{1}-y_{2}\right| \leq V\left(t, y_{1}\right)+L_{1}\left|y_{1}-y_{2}\right| .
$$

Interchanging roles of $y_{1}$ and $y_{2}$, we conclude.

Now let $t_{1}<t_{2}, y_{0} \in Q$ and $x(\cdot) \in S_{\left[t_{1}, 1\right]}^{K}\left(y_{0}\right)$ satisfy $g(x(1)) \leq V\left(t_{1}, y_{0}\right)+$ $t_{2}-t_{1}$. Since the value function is non-decreasing along feasible trajectories, we obtain

$$
V\left(t_{1}, y_{0}\right) \leq V\left(t_{2}, x\left(t_{2}\right)\right) \leq g(x(1)) \leq V\left(t_{1}, y_{0}\right)+t_{2}-t_{1} .
$$

Hence there exists $\varepsilon \in[0,1]$ such that $V\left(t_{2}, x\left(t_{2}\right)\right)=V\left(t_{1}, y_{0}\right)+\varepsilon\left(t_{2}-t_{1}\right)$. Since $V(t, \cdot)$ is $L_{1}$-Lipschitz on $Q$ for all $t \in[0,1]$, for some $\delta \in[-1,1]$,

$$
V\left(t_{2}, y_{0}\right)=V\left(t_{2}, x\left(t_{2}\right)\right)+\delta L_{1}\left|x\left(t_{2}\right)-y_{0}\right| .
$$

Hence $V\left(t_{2}, y_{0}\right)=V\left(t_{1}, y_{0}\right)+\varepsilon\left(t_{2}-t_{1}\right)+\delta L_{1}\left|x\left(t_{2}\right)-y_{0}\right|$. Then the essential boundedness of $\gamma$ yields the existence of $L_{2}$, depending only on $R$, such that $\left|V\left(t_{2}, y_{0}\right)-V\left(t_{1}, y_{0}\right)\right| \leq L_{2}\left(t_{2}-t_{1}\right)$.

Let $\left(\partial_{-}\right)_{x} V(t, x)$ denote the (partial) subdifferential of $V(t, \cdot)$ at $x$. In [5] the following subgradients were introduced for $(t, x) \in[0,1] \times K$ :

$$
\begin{gathered}
\partial^{\text {int }} V(t, x)=\overline{c o} \operatorname{Limsup}_{\left(t^{\prime}, y\right) \rightarrow(t, x)} \partial_{-} V\left(t^{\prime}, y\right) \\
y \in \operatorname{Int} K
\end{gathered}
$$

and

$$
\begin{gathered}
\partial_{x}^{i n t} V(t, x)=\overline{c o} \operatorname{Limsup}_{\left(t^{\prime}, y\right) \rightarrow(t, x)}\left(\partial_{-}\right)_{x} V\left(t^{\prime}, y\right) . \\
y \in \operatorname{Int} K
\end{gathered}
$$


Observe that $\partial^{\text {int }} V(\cdot, \cdot)$ and $\partial_{x}^{i n t} V(\cdot, \cdot)$ have closed graphs. If $V$ is locally Lipschitz on $[0,1] \times K$, then using that $\partial_{-} V\left(t^{\prime}, y\right) \subset \partial V\left(t^{\prime}, y\right)$ for all $y \in \operatorname{Int} K$ and [10, Theorem 2.5.1] we get

$$
\begin{gathered}
\partial^{\text {int }} V(t, x)=c o \operatorname{Limsup}_{\left(t^{\prime}, y\right) \rightarrow(t, x)}\left\{\nabla V\left(t^{\prime}, y\right)\right\} . \\
y \in \operatorname{Int} K
\end{gathered}
$$

By (5.1),

$$
\begin{gathered}
\operatorname{Limsup}_{\left(t^{\prime}, y\right) \rightarrow(t, x)}\left\{\nabla_{x} V\left(t^{\prime}, y\right)\right\} \subset \partial_{x}^{i n t} V(t, x) . \\
y \in \operatorname{Int} K
\end{gathered}
$$

This implies that for all $\left(p_{t}, p_{x}\right) \in \operatorname{Limsup}_{\left(t^{\prime}, y\right) \rightarrow(t, x), y \in \operatorname{Int} K}\left\{\nabla V\left(t^{\prime}, y\right)\right\}$ we have $p_{x} \in \partial_{x}^{i n t} V(t, x)$. Consequently, under assumptions of Theorem 5.1,

$$
\partial^{i n t} V(t, \bar{x}(t)) \subset \mathbb{R} \times \partial_{x}^{i n t} V(t, \bar{x}(t)) .
$$

Furthermore, for all $(t, x) \in(0,1) \times \operatorname{Int} K$, the set $\partial^{\text {int }} V(t, x)$ is equal to the generalized gradient of $V$ at $(t, x), \partial V(t, x)$.

Define the (true) Hamiltonian $H:[0,1] \times \mathbb{R}^{n} \times \mathbb{R}^{n} \rightarrow \mathbb{R}$ by

$$
H(t, x, p)=\max _{v \in F(t, x)}\langle p, v\rangle \text {. }
$$

Below when we write a.e. (almost everywhere) without making the measure precise, we always mean the Lebesgue measure on $[0,1]$. We use the notation $\partial H(t ; x, p)$ for the Clarke generalized gradient of $H(t, \cdot, \cdot)$ at $(x, p)$. Define $\widehat{V}(y)=-V(0, y)$ for $y \in K$ and $\widehat{V}(y)=+\infty$ for $y \notin K$.

Theorem 5.3. Assume $(A)$, $\left(I P_{\text {rel }}\right)$, that $g$ is locally Lipschitz and that $\gamma$ is essentially bounded. Let $\bar{x}$ be an optimal solution of the Mayer problem. Then there exist a positive finite Borel measure $\mu$ on $[0,1]$, a Borel measurable function $\nu(\cdot):[0,1] \rightarrow \mathbb{R}^{n}$ such that $\nu(t) \in N_{K}(\bar{x}(t)) \cap B \mu-$ a.e., and an absolutely continuous $p(\cdot):[0,1] \rightarrow \mathbb{R}^{n}$ such that for $\psi(t):=\int_{[0, t]} \nu(s) d \mu(s)$ for $t \in(0,1]$ the following relations are satisfied:

the Hamiltonian inclusion $\left(-p^{\prime}(t), \bar{x}^{\prime}(t)\right) \in \partial H(t ; \bar{x}(t), p(t)+\psi(t))$ a.e. in $[0,1]$, the maximum principle $\left\langle p(t)+\psi(t), \bar{x}^{\prime}(t)\right\rangle=H(t, \bar{x}(t), p(t)+\psi(t))$ a.e. in $[0,1]$, the transversality condition $p(1)+\psi(1) \in-\partial^{*} g(\bar{x}(1))$,

the sensitivity relations

$$
(H(t, \bar{x}(t), p(t)+\psi(t)),-p(t)-\psi(t)) \in \partial^{i n t} V(t, \bar{x}(t)) \text { a.e. in }[0,1]
$$

and

$$
p(0) \in \partial^{*} \widehat{V}\left(x_{0}\right)
$$

Furthermore

$$
\begin{gathered}
-p(t)-[\psi(t-), \psi(t)] \subset \partial_{x}^{i n t} V(t, \bar{x}(t)) \quad \forall t \in(0,1), \\
-p(1)-\psi(1-) \in \partial_{x}^{i n t} V(1, \bar{x}(1)), \quad-p(0)-\psi(0+) \in \partial_{x}^{i n t} V\left(0, x_{0}\right),
\end{gathered}
$$

where we have set $[\psi(t-), \psi(t)]=\{\lambda \psi(t-)+(1-\lambda) \psi(t) \mid \lambda \in[0,1]\}$. 
Recall that $\mu$ being a finite Borel measure on $[0,1]$, it is regular.

Note that the above maximum principle is normal and if $x_{0} \in \operatorname{Int} K$, then $-p(0) \in \partial_{x}^{*} V\left(0, x_{0}\right)$. Also, by the proof provided below, we could consider in Theorem 5.3 an optimal solution $\bar{x}$ of the relaxed Mayer problem.

Our proof uses several arguments of proofs in $[5,24]$, where sensitivity relations were investigated for $F$ described by a control system. We simplify some of them and deal with the differential inclusions framework and the true Hamiltonian $H$, while in $[5,24]$ the Pontriagin Hamiltonian was considered.

Assumption $\left(I P_{r e l}\right)$ is needed only to deduce all the conclusions of Theorem 3.3. Furthermore, the Hamiltonian associated to $c o F$ is equal to $H$. This and Theorem 5.1 imply that without any loss of generality we may assume

\section{$F$ has convex compact images.}

Lemma 5.4. There exists a subset $D$ of $[0,1]$ of full measure such that for all $t \in D, x \in \operatorname{Int} K$ and every $w \in F(t, x)$ we have $D_{\uparrow} V(t, x)(1, w) \geq 0$.

Proof. By [17, Theorem 2.9] we know that for a set $D \subset[0,1]$ of full measure and for all $t \in D, x \in \mathbb{R}^{n}$ and $w \in F(t, x)$, there exists $z \in S_{[t, 1]}(x)$ satisfying $z^{\prime}(t)=w$. Since $x \in \operatorname{Int} K$, for all small $h>0, z(t+h) \in K$.

The value function of Mayer's problem being nondecreasing along any feasible F-trajectory, we have $V(t+h, z(t+h))-V(t, x) \geq 0$ for all small $h>0$. Dividing this inequality by $h>0$, taking the limit when $h \rightarrow 0+$ and using the local Lipschitz continuity of $V$ we complete the proof.

For every integer $i \geq 1$ define the mapping $L_{i}:[0,1] \times \mathbb{R} \times \mathbb{R}^{n} \rightarrow \mathbb{R}$ by $L_{i}(t, \alpha, \beta)=\sup \left\{\left\langle\nabla V\left(t^{\prime}, y\right),(\alpha,-(1+\alpha) \beta)\right\rangle|y \in \operatorname{Int} K|,\left(t^{\prime}, y\right)-(t, \bar{x}(t)) \mid<\frac{1}{i}\right\}$.

In the above we only consider points $\left(t^{\prime}, y\right)$ at which $V$ is differentiable. $V$ being locally Lipschitz on $[0,1] \times K, L_{i}$ is bounded on $[0,1] \times[-1,1] \times B$. Moreover, for every $(\alpha, \beta) \in \mathbb{R} \times \mathbb{R}^{n}, L_{i}(\cdot, \alpha, \beta)$ is measurable and for all $t \in[0,1], L_{i}(t, \cdot, \cdot)$ is continuous. Consequently for all measurable mappings $\alpha:[0,1] \rightarrow[-1,1]$ and $\beta:[0,1] \rightarrow B$, the function $t \mapsto L_{i}(t, \alpha(t), \beta(t))$ is integrable on $[0,1]$. Furthermore, by (5.2),

$$
\begin{aligned}
& L_{i}(t, \alpha, \beta) \\
& \quad=\sup \left\{\langle q,(\alpha,-(1+\alpha) \beta)\rangle|y \in \operatorname{Int} K,|\left(t^{\prime}, y\right)-(t, \bar{x}(t)) \mid<\frac{1}{i}, q \in \partial V\left(t^{\prime}, y\right)\right\} .
\end{aligned}
$$

Lemma 5.5. Let $t_{0} \in[0,1), i \geq 1$. Fix measurable $\alpha:\left[t_{0}, 1\right] \rightarrow\left[-\frac{1}{i}, \frac{1}{i}\right]$ and $\beta:\left[t_{0}, 1\right] \rightarrow \frac{1}{i} B$. Consider an absolutely continuous $x:\left[t_{0}, 1\right] \rightarrow \operatorname{Int} K$ such that $\|x-\bar{x}\|_{\infty}<\frac{1}{i}$ and

$$
x^{\prime}(t) \in(1+\alpha(t))(F(t, x(t))+\beta(t)) \text { a.e. in }\left[t_{0}, 1\right] .
$$

Then for almost every $t \in\left[t_{0}, 1\right]$ we have $\frac{d}{d t} V(t, x(t))+L_{i}(t, \alpha(t), \beta(t)) \geq 0$.

Proof. Since $V$ is locally Lipschitz on $[0,1] \times K$ and $\gamma$ is essentially bounded, $V(\cdot, x(\cdot))$ is Lipschitz. Consider $D$ as in Lemma 5.4 and a measurable selection $v(t) \in F(t, x(t))$ such that $x^{\prime}(t)=(1+\alpha(t))(v(t)+\beta(t))$ a.e. Fix $t \in D$ such 
that $V(\cdot, x(\cdot))$ is differentiable at $t$ and $x^{\prime}(t)=(1+\alpha(t))(v(t)+\beta(t))$. By the mean value theorem [10, Theorem 2.3.7], for all small $h>0$ and for some $\theta_{h} \in[0,1]$, there exist

$$
p_{h} \in \partial V\left(t+h+h \theta_{h} \alpha(t), x(t)+h x^{\prime}(t)-h \theta_{h}(1+\alpha(t)) \beta(t)\right)
$$

such that

$$
\begin{aligned}
& h\left\langle p_{h},(\alpha(t),-(1+\alpha(t)) \beta(t))\right\rangle \\
& \quad=V\left(t+h(1+\alpha(t)), x(t)+h x^{\prime}(t)-h(1+\alpha(t)) \beta(t)\right)-V\left(t+h, x(t)+h x^{\prime}(t)\right) .
\end{aligned}
$$

By Lemma $5.4, D_{\uparrow} V(t, x(t))(1, v(t)) \geq 0$. Since $D_{\uparrow} V(t, x(t))(\cdot)$ is positively homogeneous, $D_{\uparrow} V(t, x(t))(1+\alpha(t),(1+\alpha(t)) v(t)) \geq 0$. Therefore

$$
\begin{aligned}
0 & \leq \liminf _{h \rightarrow 0+} \frac{V(t+h(1+\alpha(t)), x(t)+h(1+\alpha(t)) v(t))-V(t, x(t))}{h} \\
& \leq \frac{d}{d t} V(t, x(t))+\limsup _{h \rightarrow 0+}\left\langle p_{h},(\alpha(t),-(1+\alpha(t)) \beta(t))\right\rangle .
\end{aligned}
$$

This and the definition of $L_{i}$ imply that $0 \leq \frac{d}{d t} V(t, x(t))+L_{i}(t, \alpha(t), \beta(t))$.

Lemma 5.6. Let $\bar{x}(\cdot)$ be optimal for the Mayer problem. Then there exist $c_{0}>0$ and $i_{0} \geq 1$ such that for all $c \geq c_{0}$ and $i \geq i_{0}$, the quintuple $(z(\cdot) \equiv 0, y(\cdot) \equiv$ $\left.-V\left(0, x_{0}\right), x(\cdot)=\bar{x}(\cdot), \alpha(\cdot) \equiv 0, \beta(\cdot) \equiv 0\right)$ is optimal for the problem

$$
\text { minimize } g(x(1))+\int_{0}^{1} L_{i}(t, \alpha(t), \beta(t)) d t+c z(1)+y(1)
$$

over solutions of the differential inclusion

$$
\left\{\begin{array}{l}
z^{\prime}=0 \\
y^{\prime}=0, y(0) \geq \widehat{V}(x(0)) \\
x^{\prime} \in(1+\alpha(t))(F(t, x)+\beta(t)),(\alpha(t), \beta(t)) \in\left[-\frac{1}{i}, \frac{1}{i}\right] \times \frac{1}{i} B \text { a.e. } \\
d_{K}(x(t)) \leq z(t) \text { for all } t \in[0,1] \\
\left\|x(0)-x_{0}\right\| \leq 1
\end{array}\right.
$$

Proof. Let $r_{0}=\left|x_{0}\right|+1$ and $\varepsilon, L>0$ be such that the conclusion of Theorem 3.3 holds true for all measurable $(\alpha, \beta):[0,1] \rightarrow[-\varepsilon, \varepsilon] \times \varepsilon B$ and the differential inclusion

$$
x^{\prime}(t) \in(1+\alpha(t))(F(t, x(t))+\beta(t)) .
$$

By assumption (A) and the Gronwall lemma there exists $R>\|\bar{x}\|_{\infty}$ such that for all measurable $\alpha, \beta:[0,1] \rightarrow[-\varepsilon, \varepsilon] \times \varepsilon B$, any trajectory $x(\cdot)$ of $(5.8)$ with $x(0) \in B\left(x_{0}, 1\right)$ satisfies $\|x\|_{\infty} \leq R$. Let $k$ be a Lipschitz constant of $g$ on $B(0, R)$ and denote by $T_{\alpha, \beta}\left(y_{0}\right)$ the set of all trajectories of (5.8) starting at $y_{0} \in K$ and defined on $[0,1]$ and by $T_{\alpha, \beta}^{K}\left(y_{0}\right)$ the set of all $x(\cdot) \in T_{\alpha, \beta}\left(y_{0}\right)$ satisfying $x([0,1]) \subset K$. Let $i_{0} \geq 1$ be such that $\frac{1}{i_{0}}<\varepsilon$ and fix $i \geq i_{0}$, measurable $\alpha, \beta:[0,1] \rightarrow\left[-\frac{1}{i}, \frac{1}{i}\right] \times \frac{1}{i} B$ and $y_{0} \in K \cap B\left(x_{0}, 1\right), \widehat{x}(\cdot) \in T_{\alpha, \beta}\left(y_{0}\right)$. By Theorem 3.3 there exists $x \in T_{\alpha, \beta}^{K}\left(y_{0}\right)$ satisfying

$$
\|x-\widehat{x}\|_{W^{1,1}} \leq(L+1) \max _{t \in[0,1]} d_{K}(\widehat{x}(t)) .
$$


Since $g(x(1)) \leq g(\widehat{x}(1))+k|x(1)-\widehat{x}(1)|$, we deduce that for every $c \geq c_{0}:=$ $k(L+1)$

$$
\min \left\{g(x(1)) \mid x \in T_{\alpha, \beta}^{K}\left(y_{0}\right)\right\} \leq g(\hat{x}(1))+c \max _{t \in[0,1]} d_{K}(\hat{x}(t)) .
$$

Recall that $L_{i}(t, 0,0)=0$ for every $i$. To complete the proof of Lemma it is sufficient to prove the following claim: for any $y_{0} \in K \cap B\left(x_{0}, 1\right)$ we have

$$
\begin{aligned}
& g(\bar{x}(1))-V\left(0, x_{0}\right) \\
& \quad \leq \min _{x \in T_{\alpha, \beta}\left(y_{0}\right)}\left(g(x(1))+\int_{0}^{1} L_{i}(t, \alpha(t), \beta(t)) d t+c \max _{t \in[0,1]} d_{K}(x(t))-V\left(0, y_{0}\right)\right) .
\end{aligned}
$$

For this aim observe that, by (5.9), it is enough to show that

$$
g(\bar{x}(1))-V\left(0, x_{0}\right) \leq \min _{y \in T_{\alpha, \beta}^{K}\left(y_{0}\right)} g(y(1))+\int_{0}^{1} L_{i}(t, \alpha(t), \beta(t)) d t-V\left(0, y_{0}\right) .
$$

Let $x \in T_{\alpha, \beta}^{K}\left(y_{0}\right)$ be such that $g(x(1))=\min _{y \in T_{\alpha, \beta}^{K}\left(y_{0}\right)} g(y(1))$. By Theorem 3.3 we can find a sequence $x_{j}(\cdot) \in T_{\alpha, \beta}^{K}\left(y_{0}\right)$ converging uniformly to $x(\cdot)$ such that $x_{j}((0,1]) \subset \operatorname{Int} K$. Then for every integer $m \geq 1$,

$$
V\left(1, x_{j}(1)\right)-V\left(m^{-1}, x_{j}\left(m^{-1}\right)\right)=\int_{\frac{1}{m}}^{1} \frac{d}{d t} V\left(t, x_{j}(t)\right) d t .
$$

Passing to the limit we obtain $g\left(x_{j}(1)\right)-V\left(0, y_{0}\right)=\int_{0}^{1} \frac{d}{d t} V\left(t, x_{j}(t)\right) d t$. This and Lemma 5.5 imply that for all $j$ large enough

$$
g\left(x_{j}(1)\right)+\int_{0}^{1} L_{i}(t, \alpha(t), \beta(t)) d t-V\left(0, y_{0}\right) \geq 0
$$

On the other hand, since $\bar{x}$ is optimal for the Mayer problem, $g(\bar{x}(1))-$ $V\left(0, x_{0}\right)=0$. Therefore, passing to the limit when $j \rightarrow \infty$, we get

$$
g(x(1))+\int_{0}^{1} L_{i}(t, \alpha(t), \beta(t)) d t-V\left(0, y_{0}\right) \geq g(\bar{x}(1))-V\left(0, x_{0}\right)
$$

and the proof follows.

Proof of of Theorem 5.3. . Consider $i_{0} \geq 2, c_{0}$ as in Lemma 5.6 and let $R>0$ be as in the proof of Lemma 5.6. Let $M>0$ be such that for all $i \geq i_{0}$,

$$
\sup \left\{\left|L_{i}(t, \alpha, \beta)\right||t \in[0,1],| \alpha \mid \leq \frac{1}{i}, \beta \in \frac{1}{i} B\right\} \leq M .
$$

Fix any $i \geq i_{0}$ and $c \geq c_{0}$.

Then $\left(z(\cdot) \equiv 0, y(\cdot) \equiv-V\left(0, x_{0}\right), x(\cdot)=\bar{x}(\cdot), \alpha(\cdot) \equiv 0, \beta(\cdot) \equiv 0\right)$ is a minimizer for the problem (5.6)-(5.7), by Lemma 5.6. Consider the set valued $\operatorname{map} G:[0,1] \times \mathbb{R} \times \mathbb{R}^{n} \leadsto \mathbb{R}^{n+1}$ defined by

$$
\begin{gathered}
G(t, w, x):=\left\{\left(L_{i}(t, \alpha, \beta)+r,(1+\alpha)(F(t, x)+\beta)\right)|| \alpha \mid \leq \frac{1}{i},\right. \\
\left.\beta \in \frac{1}{i} B, r \in\left[0, M-L_{i}(t, \alpha, \beta)\right]\right\} .
\end{gathered}
$$


We claim that it has convex compact images. Indeed compactness follows from the compactness of $F(t, x)$ and the continuity of $L_{i}(t, \cdot, \cdot)$. To prove convexity we first observe that the set $\left\{(1,-\beta, F(t, x)+\beta) \mid \beta \in \frac{1}{i} B\right\}$ is convex. Hence also the set

$$
\Theta(t, x):=\left\{(1+\alpha,-(1+\alpha) \beta,(1+\alpha)(F(t, x)+\beta))|| \alpha \mid \leq \frac{1}{i}, \beta \in \frac{1}{i} B\right\}
$$

is convex. Observe next that

$$
\begin{aligned}
L_{i}(t, \alpha, \beta)= & \sup \left\{\left\langle\left(q_{t}, q_{x}\right),(1+\alpha,-(1+\alpha) \beta)\right\rangle-q_{t} \mid\right. \\
& \left.y \in \operatorname{Int} K,\left|\left(t^{\prime}, y\right)-(t, \bar{x}(t))\right|<1 / i,\left(q_{t}, q_{x}\right) \in \partial V\left(t^{\prime}, y\right)\right\},
\end{aligned}
$$

where $q_{t} \in \mathbb{R}$ and $q_{x} \in \mathbb{R}^{n}$. Fix any $\beta_{j} \in \frac{1}{i} B,\left|\alpha_{j}\right| \leq \frac{1}{i}, v_{j} \in F(t, x)$ for $j=1,2$ and let $\lambda \in(0,1)$. By convexity of $\Theta(t, x)$, there exist $|\alpha| \leq \frac{1}{i}$ and $\beta \in \frac{1}{i} B$ such that

$$
\begin{aligned}
\lambda(1+ & \left.\alpha_{1},-\left(1+\alpha_{1}\right) \beta_{1},\left(1+\alpha_{1}\right)\left(v_{1}+\beta_{1}\right)\right) \\
& +(1-\lambda)\left(1+\alpha_{2},-\left(1+\alpha_{2}\right) \beta_{2},\left(1+\alpha_{2}\right)\left(v_{2}+\beta_{2}\right)\right) \\
& \quad(1+\alpha,-(1+\alpha) \beta,(1+\alpha)(F(t, x)+\beta)) .
\end{aligned}
$$

Furthermore, for all $y \in \operatorname{Int} K$ satisfying $\left|\left(t^{\prime}, y\right)-(t, \bar{x}(t))\right|<1 / i$ and $\left(q_{t}, q_{x}\right) \in$ $\partial V\left(t^{\prime}, y\right)$ we have $\left\langle\left(q_{t}, q_{x}\right),(\alpha,-(1+\alpha) \beta)\right\rangle=\left\langle\left(q_{t}, q_{x}\right),(1+\alpha,-(1+\alpha) \beta)\right\rangle-q_{t}$. Therefore

$$
\begin{aligned}
\left\langle\left(q_{t}, q_{x}\right),(\alpha,-(1+\alpha) \beta)\right\rangle= & \lambda\left\langle\left(q_{t}, q_{x}\right),\left(\alpha_{1},-\left(1+\alpha_{1}\right) \beta_{1}\right)\right\rangle \\
& +(1-\lambda)\left\langle\left(q_{t}, q_{x}\right),\left(\alpha_{2},-\left(1+\alpha_{2}\right) \beta_{2}\right)\right\rangle .
\end{aligned}
$$

Hence $\left\langle\left(q_{t}, q_{x}\right),(\alpha,-(1+\alpha) \beta)\right\rangle \leq \lambda L_{i}\left(t, \alpha_{1}, \beta_{1}\right)+(1-\lambda) L_{i}\left(t, \alpha_{2}, \beta_{2}\right)$. This implies that $L_{i}(t, \alpha, \beta) \leq \lambda L_{i}\left(t, \alpha_{1}, \beta_{1}\right)+(1-\lambda) L_{i}\left(t, \alpha_{2}, \beta_{2}\right) \leq M$. Consequently $G(t, w, x)$ is convex.

Consider the following Mayer problem

$$
\operatorname{minimize} g(x(1))+w(1)+c z(1)+y(1)
$$

over trajectories of the differential inclusion

$$
\left\{\begin{array}{l}
z^{\prime}=0, \\
y^{\prime}=0, y(0) \geq \widehat{V}(x(0)) \\
\left(w^{\prime}, x^{\prime}\right) \in G(t, w, x) \\
d_{K}(x(t)) \leq z(t) \text { for all } t \in[0,1] \\
w(0)=0,\left|x(0)-x_{0}\right| \leq 1
\end{array}\right.
$$

By Lemma $5.6,\left(z(\cdot) \equiv 0, y(\cdot) \equiv-V\left(0, x_{0}\right), w(\cdot) \equiv 0, x(\cdot)=\bar{x}(\cdot)\right)$ is a minimizer of the above problem.

For every $i \geq 1$ define the Hamiltonian $H_{i}:[0,1] \times \mathbb{R} \times \mathbb{R}^{n} \times \mathbb{R} \times \mathbb{R}^{n} \rightarrow \mathbb{R}$

$$
H_{i}(t, w, x, q, p)=\max _{(a, b) \in G(t, w, x)}\langle(q, p),(a, b)\rangle,
$$

where $a \in \mathbb{R}, b \in \mathbb{R}^{n}$. Then $H_{i}(t, w, x, \cdot, \cdot)$ is positively homogeneous. Denote by $\partial^{*} H_{i}(t ; w, x, q, p)$ the limiting subdifferential of $H_{i}(t, \cdot, \cdot, \cdot, \cdot)$ at $(w, x, q, p)$ and by $\partial H_{i}(t ; w, x, q, p)$ the generalized gradient of $H_{i}(t, \cdot, \cdot, \cdot, \cdot)$ at $(w, x, q, p)$.

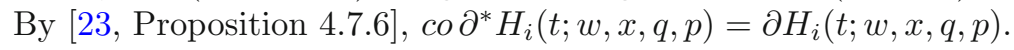


Since $H_{i}$ does not depend on $w$, we deduce that for every $(d, e, f, g) \in$ $\mathbb{R} \times \mathbb{R}^{n} \times \mathbb{R} \times \mathbb{R}^{n}$ satisfying $(d, e, f, g) \in \partial^{*} H_{i}(t ; w, x, q, p)$, we have $d=0$. Denote by $N_{e p i \widehat{V}}^{*}\left(x_{0},-V\left(0, x_{0}\right)\right)$ the limiting normal cone to the epigraph of $\widehat{V}$ at $\left(x_{0},-V\left(0, x_{0}\right)\right)$ (see [23, Definition 4.2.3 and Proposition 4.2.5]). Recall that $\partial d_{K}(x) \subset N_{K}(x) \cap B$ for all $x \in K$.

The maximum principle [23, Theorem 10.4.1] applied with $h(z, x):=$ $d_{K}(x)-z$ implies that for every $i \geq i_{0}$, there exist multipliers $\zeta_{i}, \vartheta_{i}, q_{i} \in$ $\mathbb{R}, p_{i} \in W^{1,1}\left([0,1] ; \mathbb{R}^{n}\right), \lambda_{i} \geq 0$, a positive finite regular measure $\mu_{i} \in$ $C([0,1] ; \mathbb{R})^{*}($ dual space of $C([0,1] ; \mathbb{R}))$ on $[0,1]$ and a $\mu_{i}-$ measurable selection $\nu_{i}(s) \in N_{K}(\bar{x}(s)) \cap B$ such that

$$
\lambda_{i}+\left\|p_{i}\right\|_{\infty}+\int_{[0,1]} \mu_{i}(d s)+\left|\zeta_{i}\right|+\left|\vartheta_{i}\right|+\left|q_{i}\right|>0
$$

and for $\hat{\psi}_{i}(t):=\int_{[0, t)} \nu_{i}(s) \mu_{i}(d s)$, for $t \in(0,1)$ and $\hat{\psi}_{i}(1):=\int_{[0,1]} \nu_{i}(s) \mu_{i}(d s)$ the following relations are satisfied: the adjoint inclusion

$$
p_{i}^{\prime}(t) \in \operatorname{co}\left\{-\xi \mid\left(0, \xi, 0, \bar{x}^{\prime}(t)\right) \in \partial^{*} H_{i}\left(t ; 0, \bar{x}(t), q_{i}, p_{i}(t)+\hat{\psi}_{i}(t)\right)\right\} \text { a.e. }
$$

the transversality conditions

$$
\begin{gathered}
-q_{i}=\lambda_{i},-p_{i}(1)-\hat{\psi}_{i}(1) \in \lambda_{i} \partial^{*} g(\bar{x}(1)), \quad-\zeta_{i}+\int_{[0,1]} d \mu_{i}(s)=\lambda_{i} c \\
-\vartheta_{i}=\lambda_{i}, \zeta_{i}=0,\left(p_{i}(0), \vartheta_{i}\right) \in N_{e p i \widehat{V}}^{*}\left(x_{0},-V\left(0, x_{0}\right)\right)
\end{gathered}
$$

and the maximum principle

$$
\left\langle p_{i}(t)+\hat{\psi}_{i}(t), \bar{x}^{\prime}(t)\right\rangle-\lambda_{i} L_{i}(t, 0,0)=\max _{(a, b) \in G(t, 0, \bar{x}(t))}\left\langle\left(q_{i}, p_{i}(t)+\hat{\psi}_{i}(t)\right),(a, b)\right\rangle \text { a.e. }
$$

Since $H_{i}(t, w, x, \cdot, \cdot)$ is positively homogeneous and $\gamma \in L_{+}^{\infty}([0,1])$, the adjoint inclusion (5.11) and assumptions (A) imply that there exists $\ell \in L^{1}([0,1])$ such that

$$
\left|p_{i}^{\prime}(t)\right| \leq \ell(t)\left(\left|q_{i}\right|+\left|p_{i}(t)+\hat{\psi}_{i}(t)\right|\right) \text { a.e. in }[0,1]
$$

Observe that the above transversality conditions imply that if $\lambda_{i}=0$, then $q_{i}=\vartheta_{i}=0, \mu_{i}=0, \hat{\psi}_{i}=0, p_{i}(1)=0$. Then, by $(5.12)$, also $p_{i}(\cdot)=0$, in contradiction with (5.10). Thus $\lambda_{i}>0$. Multiplying the obtained multipliers by a positive constant, we may assume that $\lambda_{i}=1$. Then

$$
\int_{[0,1]} d \mu_{i}(s)=c, \vartheta_{i}=-1, q_{i}=-1
$$

and, by [23, Definition 4.3.1], $p_{i}(0) \in \partial^{*} \widehat{V}\left(x_{0}\right)$.

Consequently $\left\{\hat{\psi}_{i}(\cdot)\right\}_{i \geq i_{0}}$ is uniformly bounded and therefore $\left\{\left|p_{i}(1)\right|\right\}_{i \geq i_{0}}$ is uniformly bounded. Hence, by (5.12) and the Gronwall lemma, also $\left\{p_{i}(\cdot)\right\}_{i \geq i_{0}}$ is uniformly bounded. This implies that for some $k(\cdot) \in L_{+}^{\infty}([0,1])$, for all $i \geq i_{0}$ and for a.e. $t \in[0,1]$ we have $\left|p_{i}^{\prime}(t)\right| \leq k(t)$. 
Let $N$ be such that $\left\|p_{i}\right\|_{\infty} \leq N$ for all $i$. Observe next that for all $i \geq 2$ we have $1+\alpha>0$ whenever $|\alpha| \leq \frac{1}{i}$. By the very definition of $G$ for every $q \in[-2,0]$,

$$
\begin{aligned}
& H_{i}(t, w, x, q, p) \\
& \quad=\max _{|\alpha| \leq \frac{1}{i}, \beta \in \frac{1}{i} B}\left((1+\alpha) H(t, x, p)+\langle p,(1+\alpha) \beta\rangle+q L_{i}(t, \alpha, \beta)\right) \\
& \quad=H(t, x, p)+\max _{|\alpha| \leq \frac{1}{i}, \beta \in \frac{1}{i} B}\left(\alpha H(t, x, p)+\langle p,(1+\alpha) \beta\rangle+q L_{i}(t, \alpha, \beta)\right) .
\end{aligned}
$$

Define

$$
\Phi_{i}(t, x, q, p)=\max _{|\alpha| \leq \frac{1}{i}, \beta \in \frac{1}{i} B}\left(\alpha H(t, x, p)+\langle p,(1+\alpha) \beta\rangle+q L_{i}(t, \alpha, \beta)\right) .
$$

By assumptions (A), continuity of $L_{i}(t, \cdot, \cdot)$ and the very definition of $\Phi_{i}$ there exist $\ell_{i}(t)>0$ such that $\lim _{i \rightarrow \infty} \ell_{i}(t)=0$ and for a.e. $t \in[0,1], \Phi_{i}(t, \cdot, \cdot, \cdot)$ is $\ell_{i}(t)$-Lipschitz on the set $B(\bar{x}(t), 1) \times[-2,0] \times(N+1) B$. Let $\stackrel{\circ}{B}_{2 n+2}$ and $B_{2 n+2}$ denote respectively open and closed unit balls in $\mathbb{R}^{2 n+2}$ and $B_{2 n}$ the closed unit ball in $\mathbb{R}^{2 n}$.

Consider any $(d, e, f, g) \in \mathbb{R} \times B(\bar{x}(t), 1) \times(-2,0) \times(N+1) \stackrel{\circ}{B}(0,1)$ such that $H_{i}(t, \cdot, \cdot, \cdot, \cdot)$ is differentiable at $(d, e, f, g)$. Then, by the definition of the generalized gradient in [10, p. 27],

$$
\frac{\partial H_{i}}{\partial(w, x, q, p)}(t, d, e, f, g) \in\{(0, \xi, 0, \tau) \mid(\xi, \tau) \in \partial H(t ; e, g)\}+\ell_{i}(t) B_{2 n+2} \text {. }
$$

This and [10, Theorem 2.5.1] imply that for any $(d, e, f, g) \in \mathbb{R} \times B(\bar{x}(t), 1) \times$ $(-2,0) \times(N+1) \stackrel{\circ}{B}$

$$
\partial H_{i}(t ; d, e, f, g) \subset\{(0, \xi, 0, \tau) \mid(\xi, \tau) \in \partial H(t ; e, g)\}+\ell_{i}(t) B_{2 n+2} .
$$

From (5.13) and the adjoint inclusion (5.11) we deduce that

$$
\left(-p_{i}^{\prime}(t), \bar{x}^{\prime}(t)\right) \in \partial H\left(t ; \bar{x}(t), p_{i}(t)+\hat{\psi}_{i}(t)\right)+\ell_{i}(t) B_{2 n} \text { a.e. in }[0,1] .
$$

Since the maximum of the scalar product $\left\langle\left(-1, p_{i}(t)+\hat{\psi}_{i}(t)\right),(a, b)\right\rangle$ over $(a, b) \in$ $G(t, 0, \bar{x}(t))$ is attained at $\left(0, \bar{x}^{\prime}(t)\right)$ for a.e. $t \in[0,1]$, we obtain

$$
-L_{i}(t, \alpha, \beta)+\left\langle p_{i}(t)+\hat{\psi}_{i}(t),(1+\alpha)(v+\beta)\right\rangle \leq\left\langle p_{i}(t)+\hat{\psi}_{i}(t), \bar{x}^{\prime}(t)\right\rangle
$$

for all $|\alpha| \leq \frac{1}{i}, \beta \in \frac{1}{i} B, v \in F(t, \bar{x}(t))$ and for almost every $t \in[0,1]$. Taking $\alpha=0$ and $\beta=0$ we get

$$
\left\langle p_{i}(t)+\hat{\psi}_{i}(t), \bar{x}^{\prime}(t)\right\rangle=H\left(t, \bar{x}(t), p_{i}(t)+\hat{\psi}_{i}(t)\right) \text { a.e. in }[0,1] .
$$

Taking $v=\bar{x}^{\prime}(t)$ we deduce that for all $|\alpha| \leq \frac{1}{i}, \beta \in \frac{1}{i} B$,

$$
-L_{i}(t, \alpha, \beta)+\left\langle p_{i}(t)+\hat{\psi}_{i}(t), \alpha \bar{x}^{\prime}(t)+(1+\alpha) \beta\right\rangle \leq 0 \text {. a.e. in }[0,1] \text {. }
$$

From (5.15) and (5.16) it follows that for a.e. $t \in[0,1]$,

$$
\left\langle\left(H\left(t, \bar{x}(t), p_{i}(t)+\hat{\psi}_{i}(t)\right),-p_{i}(t)-\hat{\psi}_{i}(t)\right),(\alpha,-(1+\alpha) \beta)\right\rangle \leq L_{i}(t, \alpha, \beta) .
$$

The above inequality, the definition of $L_{i}$ and the separation theorem imply

$$
\begin{aligned}
& \left(H\left(t, \bar{x}(t), p_{i}(t)+\hat{\psi}_{i}(t)\right),-p_{i}(t)-\hat{\psi}_{i}(t)\right) \\
& \quad \in \overline{c o}\left\{\partial V\left(t^{\prime}, y\right)|y \in \operatorname{Int} K,|\left(t^{\prime}, y\right)-(t, \bar{x}(t)) \mid<1 / i\right\} \text { a.e. in }[0,1] .
\end{aligned}
$$


Since $\hat{\psi}_{i}$ is of bounded variation, it has at most a countable number of discontinuities. Define $\psi_{i}(t)=\int_{[0, t]} \nu_{i}(s) \mu_{i}(d s), \psi_{i}(0)=0$. Then $\psi_{i}$ satisfies $(5.14)$, (5.15) and (5.17) with $\hat{\psi}_{i}$ replaced by $\psi_{i}$. Moreover $\psi_{i}(1)=\hat{\psi}_{i}(1)$.

Taking subsequences and using the same notations we may assume that for a positive finite regular measure $\mu \in C([0,1] ; \mathbb{R})^{*}$ and $p \in W^{1,1}\left([0,1] ; \mathbb{R}^{n}\right)$, $p_{i}^{\prime}$ converge weakly in $L^{1}$ to $p^{\prime}$ and $p_{i}$ converge uniformly to $p$, while $\mu_{i}$ converge to $\mu$ in the weak-* topology. By the Helly selection theorem, taking again a subsequence and keeping the same notation, we may assume that $\psi_{i}$ converge pointwise to a function $\psi$ of bounded total variation.

Define $\eta_{i} \in C\left([0,1] ; \mathbb{R}^{n}\right)^{*}$ by $\eta_{i}(d t)=\nu_{i}(t) \mu_{i}(d t)$. By [23, Proposition 9.2.1] there exists a Borel measurable selection $\nu(s) \in N_{K}(\bar{x}(s)) \cap B$ such that for $\eta \in C\left(0,1 ; \mathbb{R}^{n}\right)^{*}$ defined by $\eta(d t)=\nu(t) \mu(d t)$ a subsequence of $\eta_{i}$ (again denoted by $\eta_{i}$ ) converge to $\eta$ weakly-*. Then for all $t \in(0,1], \psi(t)=$ $\int_{[0, t]} \nu(s) \mu(d s)$. Thus $\psi$ is continuous from the right on $(0,1)$. Since $\partial H(t ; \cdot, \cdot)$ is upper semicontinuous, by the same arguments as in the proof of the convergence theorem [2, p.273], passing to the limit in (5.14), (5.15), (5.17) with $\hat{\psi}_{i}$ replaced by $\psi_{i}$, we deduce that $p$ and $\psi$ satisfy the Hamiltonian inclusion, the maximum principle, the transversality conditions and the sensitivity relations (5.3), (5.4).

Finally, from the inclusion $\partial^{\text {int }} V(t, \bar{x}(t)) \subset \mathbb{R} \times \partial_{x}^{\text {int }} V(t, \bar{x}(t))$, we get

$$
-p(t)-\psi(t) \in \partial_{x}^{\text {int }} V(t, \bar{x}(t)) \quad \text { a.e. in }(0,1) .
$$

Since $t \leadsto \partial_{x}^{i n t} V(t, \bar{x}(t))$ is upper semicontinuous, has convex compact images and $\psi$ is of bounded variation and continuous from the right on $(0,1)$, we obtain (5.5). Taking limits in (5.18) when $t \rightarrow 0+$ and $t \rightarrow 1$ - we end the proof of Theorem 5.3.

\section{References}

[1] Aubin, J.P., Cellina, A.: Differential Inclusions. Springer, Berlin (1984)

[2] Aubin, J.-P., Frankowska, H.: Set-Valued Analysis. Birkhäuser, Boston (1990)

[3] Bettiol, P., Bressan, A., Vinter, R.B.: On trajectories satisfying a state constraint: $W^{1,1}$ estimates and counter-examples. SIAM J. Control Optim. 48, 4664-4679 (2010)

[4] Bettiol, P., Frankowska, H., Vinter, R.B.: $L^{\infty}$ estimates on trajectories confined to a closed subset. J. Differ. Equ. 252, 1912-1933 (2012)

[5] Bettiol, P., Vinter, R.B.: Sensitivity interpretations of the co-state variable for optimal control problems with state constraints. SIAM J. Control Optim. 48, 3297-3317 (2010)

[6] Cannarsa, P., Frankowska, H.: Quelques charactérisations des trajectoires optimales dans la théorie de contrôle. CRAS, Série 1, Paris 310, 179-182 (1990)

[7] Cannarsa, P., Frankowska, H.: Some characterizations of optimal trajectories in control theory. SIAM J. Control Optim. 29, 1322-1347 (1991) 
[8] Cernea, A., Frankowska, H.: A connection between the maximum principle and dynamic programming for constrained control problems. SIAM J. Control Optim. 44, 673-703 (2006)

[9] Cernea, A., Frankowska, H.: A note on the value function for constrained control problems. Syst. Control Lett. 55, 21-26 (2006)

[10] Clarke, F.H.: Optimization and Nonsmooth Analysis. Wiley, New York (1983)

[11] Clarke, F.H., Vinter, R.B.: The relationship between the maximum principle and dynamic programming. SIAM J. Control Optim. 25, 1291-1311 (1987)

[12] Dubovitskii, A.Y., Milyutin, A.A.: Extremal problems with constraints. USSR Comput. Math. Math. Phys. 5, 1-80 (1965)

[13] Filippov, A.F.: Classical solutions of differential equations with multivalued right-hand side. SIAM J. Control Optim. 5, 609-621 (1967)

[14] Frankowska, H.: The maximum principle for an optimal solution to a differential inclusion with end point constraints. SIAM J. Control Optim. 25, 145-157 (1987)

[15] Frankowska, H., Mazzola, M.: Optimal synthesis and normality of the maximum principle for optimal control problems with pure state constraints. In: Proceedings of 9th IEEE International Conference on Control and Automation (ICCA), pp. 945-950, Santiago, Chile, December 19-21, 2011

[16] Frankowska, H., Mazzola, M.: Discontinuous solutions of Hamilton-JacobiBellman equation under state constraints. Calculus Var. Partial Differ. Equ. 1-23 (2012). doi:10.1007/s00526-012-0501-8

[17] Frankowska, H., Plaskacz, S., Rzeżuchowski, T.: Measurable viability theorems and the Hamilton-Jacobi-Bellman equation. J. Differ. Equ. 116, 265-305 (1995)

[18] Frankowska, H., Rampazzo, F.: Filippov's and Filippov-Wazewski's theorems on closed domains. J. Differ. Equ. 161, 449-478 (2000)

[19] Gamkrelidze, R.V.: Optimal processes with bounded phase coordinates. Izv. Akad. Nauk, USSR, Sec. Mat. 24, 315-356 (1960)

[20] Soner, H.M.: Optimal control with state-space constraints. SIAM J. Control Optim. 24, 552-561 (1986)

[21] Subbotina, N.N.: The maximum principle and the superdifferential of the value function. Problems Control Inf. Theory/Problemy Upravlen. Teor. Inform. 18, 151-160 (1989)

[22] Tamzali-Lafond, M.: Variational inclusions under state constraints. SIAM J. Control Optim. 42, 342-362 (2003)

[23] Vinter, R.B., Optimal Control. Birkäuser, Boston (2000)

[24] Vinter, R.B.: New results on the relationship between dynamic programming and the maximum principle. Math. Control Signal Syst. 1, 97-105 (1988) 
Hélène Frankowska

CNRS and Institut de Mathématiques de Jussieu

Université Pierre et Marie Curie

case 247, 4 place Jussieu

75252 Paris

France

e-mail: frankowska@math.jussieu.fr

Marco Mazzola

Institut de Mathématiques de Jussieu

Université Pierre et Marie Curie

case 247, 4 place Jussieu

75252 Paris

France

e-mail: m.mazzola7@campus.unimib.it

Received: 31 December 2011.

Accepted: 10 July 2012. 